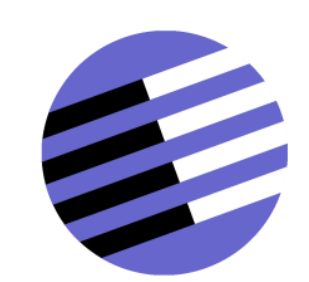

GOVERNANCE AND THE EFFICIENCY

OF ECONOMIC SYSTEMS

G ES Y

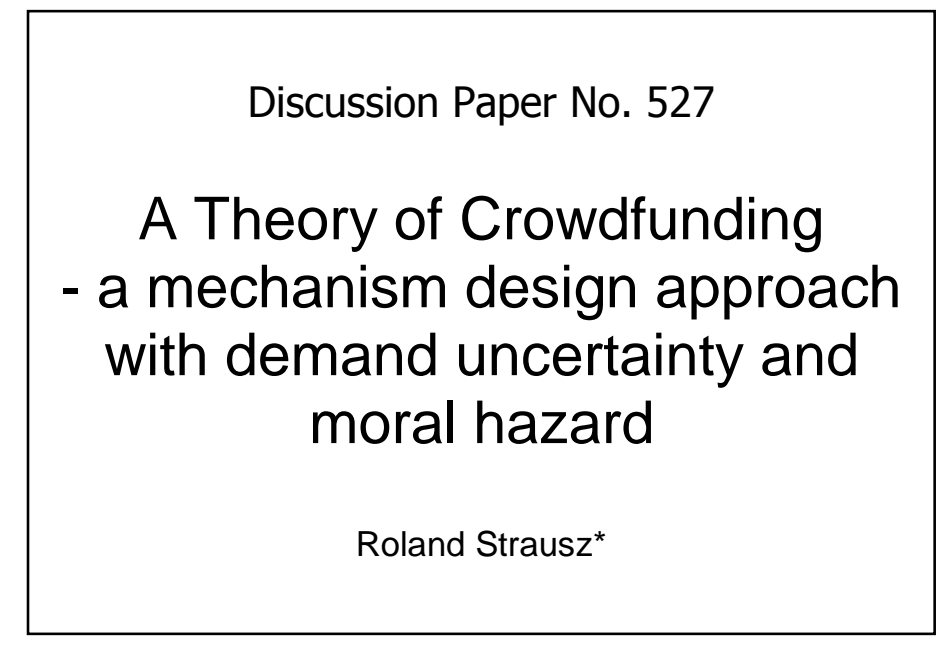

* Humboldt University of Berlin

Financial support from the Deutsche Forschungsgemeinschaft through SFB/TR 15 is gratefully acknowledged. 


\title{
A Theory of Crowdfunding \\ - a mechanism design approach with demand uncertainty and moral hazard
}

\author{
Roland Strausz* \\ This version: November 14, 2015
}

\begin{abstract}
Crowdfunding provides the innovation that, before the investment, entrepreneurs contract with consumers. Under demand uncertainty, this improves a screening for valuable projects. Entrepreneurial moral hazard threatens this benefit. Focusing on the trade-off between value screening and moral hazard, the paper characterizes optimal mechanisms. Current crowdfunding schemes reflect their salient features. Efficiency is sustainable only if returns exceed investment costs by a margin reflecting the degree of moral hazard. Constrained efficient mechanisms exhibit underinvestment. Crowdfunding blurs the distinction between finance and marketing, but complements rather than substitutes traditional entrepreneurial financing. As a screening tool for valuable projects, crowdfunding unambiguously promotes social welfare.
\end{abstract}

Keywords: Crowdfunding, finance, marketing, demand uncertainty, moral hazard

${ }^{*}$ Humboldt-Universität zu Berlin, Institute for Economic Theory 1, Spandauer Str. 1, D-10178 Berlin (Germany), strauszr@wiwi.hu-berlin.de. I thank Helmut Bester, Simon Board, Tilman Börgers, Peter Cramton, Willy Fuchs, Sebastian Kodritsch, Daniel Krähmer, Nicolas Lambert, Matthias Lang, Johannes Maier, Moritz Meyer-ter-Vehn, Steven Tadelis, Georg Weizsäcker, and audiences at EUI Florence, Stanford, UCLA, Berkeley and Tutzing for helpful discussions. I further thank Tilman Fries for research assistance. Financial support by the DFG (German Science Foundation) under SFB/TR-15 and SFB649, as well as by the (currently) 35 crowdfunders who pledged in my Kickstarter campaign "An Economic Theory of Crowdfunding" is gratefully acknowledged. 


\section{Introduction}

Crowdfunding has, in recent years, attracted much attention as a new mode of entrepreneurial financing: through the internet many individuals - the crowd - provide funds directly to the entrepreneur. ${ }^{1}$ In the narrower context of reward crowdfunding, this crowd consists of the very consumers whom the entrepreneur intends to target with her product.

Hence, crowdfunding provides the economic innovation that, in contrast to traditional modes of financing, entrepreneurs can contract with their future consumers already before the investment decision. Focusing on this ability, this paper argues that crowdfunding leads to a more effective screening of valuable projects. The screening benefit obtains, because the contract can condition the entrepreneur's investment decision on the consumers' reports about their demand. ${ }^{2}$ Before illustrating this effect in a concrete example, we first describe how reward crowdfunding works in practice. The description clarifies the features by which crowdfunding schemes elicit consumers' private information and thereby achieve a more effective screening of projects.

Attracting pledges of more than 2 billion dollars, the most successful crowdfunding platform to date is Kickstarter. ${ }^{3}$ It implements crowdfunding as follows. First, the entrepreneur describes her project, consisting of the following three elements: 1) a description of the reward to the consumer, which is typically the entrepreneur's final product; 2) a "pledge level" $p$; and 3) a "target level" $T$. After describing these elements, a number, say $\tilde{n}$, of consumers pledge contributions. If the sum of pledges exceed the target level, i.e. if $\tilde{n} \cdot p \geq T$, the entrepreneur receives the contribution $p$ from each of the $\tilde{n}$ pledging consumers and in return delivers to each of them the

\footnotetext{
${ }^{1}$ Time Magazine (2010) lists crowdfunding as one of the "Best Inventions of 2010", while The Economist (2012) reports that the "talk of crowdfunding as a short-lived fad has largely ceased". On the policy side, the JOBS Act from 2012 and SEC (2015) are meant to boost crowdfunding in the US by clarifying its legal and regulatory foundations. Even though the word "crowdfunding" dates only from 2006, the practise itself goes back much further and has, for instance, been used in the context of publishing.

${ }^{2}$ Also Ellman and Hurkens (2014) point out this conditioning feature of crowdfunding but, abstracting from moral hazard and restricting to ad hoc mechanisms, focus on commitment effects and potential benefits for price discrimination.

${ }^{3}$ See www.kickstarter.com and www.kickstarter.com/help/stats for statistics.
} 
promised reward. If the pledged contributions lie below the target level, $\tilde{n} \cdot p<T$, then the project is cancelled; consumers withdraw their pledges and the entrepreneur has no obligations towards them. Hence, given a specified reward, the pair $(p, T)$ defines the crowdfunding scheme.

For an illustration of how a crowdfunding scheme $(p, T)$ screens for valuable projects, consider a "crowd" of only a single representative consumer. ${ }^{4}$ Suppose that the consumer's willingness to pay for the good is either high, $v_{h}=4$, or low, $v_{l}=0$, each with probability $1 / 2$. Let $I=3$ represent the development costs before the good can be produced (with no further production costs). The project, therefore, has a positive value of +1 in the state $v_{h}$, a negative value -3 in the state $v_{l}$ and, without observing the state $v$, the project has the negative value -1 in expectation.

Hence, even if the entrepreneur had the required cash, she would not invest if she cannot learn the consumer's valuation. A venture capitalist reviewing the entrepreneur's business plan faces the same problem. The crowdfunding scheme $(p, T)=(4,4)$, however, elicits the consumer's private information naturally and leads to an investment only in state $v_{h}$. Indeed, facing the scheme $(p, T)=(4,4)$, only the consumer with a high value $v_{h}=4$ considers it optimal to pledge, and, hence, the investment is triggered only in the high state. The scheme therefore induces an efficient outcome and, moreover, allows the entrepreneur to extract the entire surplus.

The example not only illustrates the main efficiency effect of crowdfunding, but also identifies the three ingredients that are crucial for generating it: 1) the presence of fixed development costs; 2) uncertainty about whether the demand of consumers is large enough to recover these costs; and 3) a trigger level that enables conditional investment. The first two ingredients are defining features of entrepreneurial financing. The third ingredient is the defining feature of a so-called "all-or-nothing" reward crowdfunding platform such as Kickstarter. ${ }^{5}$

\footnotetext{
${ }^{4} \mathrm{~A}$ single agent illustrates well the main efficiency property of crowdfunding. but hides its other effective properties such as mitigating strategic uncertainty and coordination problems.

${ }^{5}$ Platforms using "all-or-nothing" pledge schemes are, for instance, Kickstarter, Sellaband, and PledgeMusic. The "keep-what-you-raise" model, where pledges are triggered even if the target level is not reached seem more popular for platforms that focus on non-profit projects (e.g. GoFundMe). Kickstarter (www.kickstarter.com/help/stats) reports that less than $40 \%$ of the projects meet the trigger level, which confirms that the trigger level plays a crucial role.
} 
Crowdfunding, however, also seems to exhibit an important economic disadvantage as compared to more traditional modes of financing. More specifically, its replacement of financial intermediaries as investors by an uncoordinated crowd raises important concerns about entrepreneurial moral hazard. ${ }^{6}$ Economic theory provides clear efficiency arguments in favor of a specialized financial intermediary. In particular, Diamond (1984) points out that by coordinating investment through a single financial intermediary, free-riding problems associated with monitoring the borrower's behavior are circumvented. Indeed, monitoring to limit moral hazard seems especially important for entrepreneurial financing. Entrepreneurs are typically new players in the market, who, in contrast to well-established firms, have not yet had the ability to build up a reputation to demonstrate their trustworthiness. ${ }^{7}$

An analysis of crowdfunding without an explicit consideration of moral hazard seems therefore lopsided. As it turns out, this is even more so, because the crowdfunding scheme's reduction in demand uncertainty interacts with the moral hazard problem: an elimination of demand uncertainty intensifies moral hazard. Hence, in the presence of both demand uncertainty and moral hazard, a non-trivial trade-off concerning the informativeness of optimal mechanisms results.

Using the generalized mechanism design framework of Myerson (1982), we explicitly address this trade-off. More generally, we characterize mechanisms that optimally address the problem of both demand uncertainty and moral hazard. Myerson's generalized framework assumes the presence of a mediator who coordinates the communication between economic agents. One insight from our analysis is that the crowdfunding platform plays exactly the role of a mediator in the sense of Myerson.

Next to this institutional insight, our characterization of optimal mechanisms and their comparison to current crowdfunding platforms yield the following additional insights: 1) Optimal mechanisms reflect reward-based crowdfunding schemes in that crowdfunders do not obtain a monetary return from funding the entrepreneur. 2) Optimal mechanisms reflect all-or-nothing reward crowdfunding schemes in that they condition the entrepreneur's investment decision on the sum of reported con-

\footnotetext{
${ }^{6}$ Mollick (2014) considers the funding of ventures "without standard financial intermediaries" as a defining feature of crowdfunding, while Agrawal et al. (2014) stress moral hazard in crowdfunding.

${ }^{7}$ Footnotes 11 to 13 discuss actual cases of moral hazard in crowdfunding campaigns.
} 
sumer valuations. 3) Optimal mechanisms achieve first-best efficient outcomes only if the ex ante expected returns of the project exceed the entrepreneur's ex ante expected capital costs by a margin that is proportional to the threat of moral hazard. 4) Constrained efficient crowdfunding schemes display underinvestment and not overinvestment, which is consistent with an all-or-nothing reward crowdfunding scheme with a target level that exceeds the entrepreneur's investment costs. 5) Optimal mechanisms defer payments to the entrepreneur in order to reduce the threat of moral hazard, a feature which is reflected by some crowdfunding platforms such as PledgeMusic. ${ }^{8}$ 6) Optimal mechanisms resolve demand uncertainty only partially, because the moral hazard problem interacts with the reduction in demand uncertainty. Few crowdfunding schemes use deferred payments explicitly and none seem to limit the entrepreneur's information about demand uncertainty actively. This suggests that current crowdfunding schemes deal with moral hazard suboptimally. Yet, by considering an extension in which the mechanism is unable to reach all potential consumers - which reflect current crowdfunding environments more appropriately, we argue that current crowdfunding platforms use deferred payments implicitly.

The rest of the paper is organized as follows. The next section discusses the related literature. Section 3 introduces the setup and takes an intuitive approach that identifies the main trade-offs. Section 4 sets up the problem as one of mechanism design. Section 5 characterizes (constrained) efficient mechanisms. Section 6 relates optimal mechanisms to real-life crowdfunding mechanisms and examines extensions. Section 7 concludes. All formal proofs are collected in the appendix.

\section{Related literature}

Being a relatively new phenomenon, the economic literature on crowdfunding is small but growing. Concerning crowdfunding's economic underpinnings, Agrawal et al. (2014) highlight the main issues. They emphasize entrepreneurial moral hazard and mention that crowdfunding can reduce demand uncertainty. Belleflamme et al. (2015) survey current crowdfunding platforms and also point out the use of crowdfunding

\footnotetext{
${ }^{8}$ PledgeMusic explains the use of deferred payments to prevent fraud on its webpage http: //www.pledgemusic.com/blog/220-preventing-fraud (last retrieved 2 November 2015.)
} 
"for market testing under uncertain aggregate demand."

Focusing on price-discrimination, Cornelli (1996) considers a monopolistic firm that, after an initial investment, faces privately informed consumers and characterizes its optimal selling procedure. She explicitly shows that the actual composition of aggregate contributions matters rather than the sum of aggregate contributions (see also Barbieri and Malueg, 2010). As a result, crowdfunding schemes cannot deal with the price-discrimination problem optimally. Belleflamme et. al (2014), Ellman and Hurkens (2014), and Chang (2015), however, point out that, even though suboptimal, crowdfunding schemes help firms to price discriminate consumers to some degree. These paper subsequently identify the features of specific crowdfunding schemes that are helpful in this respect. ${ }^{9}$

With respect to this literature, the current paper emphasizes that crowdfunding schemes exhibit a crucial economic benefit unrelated to price discrimination. In particular, this paper stresses the role of crowdfunding in "project-value screening", where the screening focuses on identifying differences in the aggregate valuation of consumers, rather than its role in "consumer-specific-screening", where the screening focuses on identifying differences between the individual valuations of consumers and which is the primary goal of price discrimination.

Identifying these two distinctive roles of crowdfunding is also crucial for properly evaluating the welfare properties of crowdfunding schemes and addressing potential regulatory questions: project-value screening univocally benefits both welfare and consumers, whereas the use of crowdfunding as a tool for price discrimination has, at best, ambiguous effects on welfare and consumers (e.g. Bergemann et. al. 2015).

While there is little work in economics and finance that focuses on the firm's ability to screen for valuable projects by addressing consumers directly, the marketing literature explicitly addresses this issue in its subfield of market research, focusing on consumer surveys and product testing (e.g. Lauga and Ofek 2009). Ding (2007) however points out that marketing research relies on voluntary, non-incentivized reporting by consumers. He emphasizes that consumers need to be given explicit incentives for revealing their information truthfully. With respect to this literature, the current

\footnotetext{
${ }^{9}$ Also Hakenes and Schlegel (2015) restrict attention to specific crowdfunding schemes although they do not focus on price discrimination but focus on costly information acquisition.
} 
paper clarifies that crowdfunding schemes provide explicit incentives for truthtelling naturally.

Empirical studies of crowdfunding aim at identifying the crucial features of crowdfunding projects. Studies such as Agrawal et al. (2011) and Mollick (2014) focus on the geographic origin of consumers relative to the entrepreneur, while Kuppuswamy and Bayus (2013) examine the role of social information for the project's success. Focusing on investment-based crowdfunding, Hildebrand, et al. (2013) identifies an increased problem of moral hazard. Ordanini et al. (2011) present a marketing-based case study on crowdfunding and also note that crowdfunding blurs the boundaries between marketing and finance.

\section{Crowdfunding and the Information Trade-off}

This section introduces the framework. It considers an entrepreneur, who can, prior to her investment decision, directly interact with privately informed consumers about whether they value the product. We first model and discuss the role of demand uncertainty, and subsequently introduce the problem of moral hazard.

The entrepreneur. We consider a penniless entrepreneur, who needs an upfront investment of $I>0$ from investors to develop her product. After developing it, the entrepreneur can produce the good at some marginal cost $c \in[0,1)$. The entrepreneur is crucial for realizing the project and cannot sell her idea to outsiders. We normalize interest rates to zero.

The crowd. We consider a total of $n$ consumers and denote a specific consumer by the index $i=1, \ldots, n$. A consumer $i$ either values the good, $v_{i}=1$, or not, $v_{i}=$ $0 .{ }^{10}$ Hence, the $n$-dimensional vector $v=\left(v_{1}, \ldots, v_{n}\right) \in V \equiv\{0,1\}^{n}$ represents the valuation profile of the consumers. We let $\pi(v)$ denote its corresponding probability. As a result, the number of consumers with value $v=1$, which we express by $n_{1}$, is:

$$
\operatorname{Pr}\left\{n_{1}\right\} \equiv \sum_{\left\{v: \sum_{i} v_{i}=n_{1}\right\}} \pi(v) .
$$

\footnotetext{
${ }^{10}$ The binary structure ensures that demand uncertainty expresses itself only concerning the question whether the entrepreneur should invest without affecting actual pricing decisions. It clarifies that the model's driving force is not price discrimination. Section 6.4 discusses this in more detail.
} 
Since the marginal costs $c$ are smaller than 1 , we can take $n_{1}$ as the potential demand of the entrepreneur's good. Its randomness expresses the demand uncertainty.

Investing without demand uncertainty. Consider as a benchmark the case of perfect information, where the realized demand $n_{1}$ is observable so that the investment decision can directly condition on it. It is socially optimal that the entrepreneur invests if the project's revenue, $n_{1}$, covers the costs of production $I+n_{1} c$, i.e. if

$$
n_{1} \geq \bar{n} \equiv \frac{I}{1-c} \text {. }
$$

In this case, the project generates an ex ante expected aggregate surplus of

$$
S^{*}=\sum_{n_{1}=\bar{n}}^{n} \operatorname{Pr}\left\{n_{1}\right\}\left[(1-c) n_{1}-I\right] .
$$

Note that by investing whenever $n_{1} \geq \bar{n}$ and, subsequently, selling the good at a price $p=1$, the entrepreneur can appropriate the full surplus. Given that the entrepreneur obtains the funds, this behavior represents her optimal strategy. Anticipating the entrepreneur's optimal behavior, a competitive credit market is willing to lend the amount $I$ at the normalized interest rate of zero. Hence, perfect information yields an efficient outcome.

Investing with demand uncertainty. Next consider the setup with demand uncertainty, i.e. the entrepreneur must decide to invest $I$ without knowing $n_{1}$ if she wants to sell the good at some price $p$. If she does invest, it clearly remains optimal to sell the good at a price $p=1$. Hence, expected profits from investing are

$$
\bar{\Pi}=\left(\sum_{n_{1}=0}^{n} \operatorname{Pr}\left\{n_{1}\right\}(1-c) n_{1}\right)-I .
$$

It is therefore profitable to invest only if $\bar{\Pi} \geq 0$. Even though the price $p=1$ does not leave any consumer rents, the entrepreneur's decision to invest leads either to under- or over-investment. For parameter constellations such that $\bar{\Pi}<0$, the entrepreneur will not invest and, hence, under-investment results (because the good is not produced for any $n_{1}>\bar{n}$, where it would be efficient to produce). For the parameter constellation $\bar{\Pi} \geq 0$, the entrepreneur does invest $I$, but this implies overinvestment (because she produces the good also when it turns out that $n_{1}<\bar{n}$ ).

Crowdfunding. We next consider the case of demand uncertainty but with an all-or-nothing reward-based crowdfunding scheme for consumers. This means that 
the entrepreneur commits to a contract pair $(p, T)$. As explained in the introduction, its interpretation is that if at least $T / p$ consumers make a pledge so that the total amount of pledged funds, $P$, exceeds $T$, then the entrepreneur obtains $P$, invests, and produces a good for each consumer who pledged. If the total amount of pledges $P$ falls short of $T$, then the pledges are not triggered and the entrepreneur does not invest.

It is straightforward to see that crowdfunding enables the entrepreneur to extract the maximum aggregate surplus $S^{*}$ and, thereby, achieve an efficient outcome. Indeed, for any $p \in(0,1]$, it is optimal for the consumer to pledge $p$ if and only if $v=1$. As a result, exactly $n_{1}$ consumers sign up so that the sum of pledges equals $P=n_{1} p$. Hence, the project is triggered whenever $T \leq n_{1} p$. We conclude that the crowdfunding scheme $(p, T)$ with $p \in(0,1]$ yields the entrepreneur an expected profit

$$
\Pi^{c}(p, T)=\sum_{n_{1}=T / p}^{n} \operatorname{Pr}\left\{n_{1}\right\}[(1-c) p-I] .
$$

Price $p=1$ and target level $T=\bar{n}$ maximize these profits, enabling the entrepreneur to extract the associated expected surplus of $S^{*}$ and yielding an efficient outcome.

In comparison to the single consumer example of the introduction, it is worthwhile to point out two additional features of the crowdfunding scheme. First, even without any active coordination between consumers, it circumvents any potential coordination problems. This is because of the schemes second feature: it eliminates any strategic uncertainty concerning both the behavior and the private information of other consumers. In other words, the scheme's conditional pledge system leads to a game between the consumers, in which it is a (weakly) dominant strategy for each individual consumer $i$ to pledge if and only if $v_{i}=1$.

Moral hazard. The setup until now abstracted from any problems of moral hazard. Consumers are sure to obtain the good as promised if their pledge is triggered. In practice, consumers may however worry about whether the entrepreneur will in the end deliver a good that meets the initial specifications, or whether they will receive some good at all.

We capture the problem of moral hazard by assuming that, after the entrepreneur obtains the money from the crowdfunding platform, she can "make a run" for it with 
a share $\alpha \in[0,1]$. When the entrepreneur "runs", she does not incur any investment or production costs and consumers do not obtain their valuable goods. The parameter $\alpha$ measures the weakness of the institutional environment to prevent moral hazard. For the extreme $\alpha=0$, there is effectively no moral hazard, whereas for the extreme, $\alpha=1$, the principal can keep all the pledges without incurring any costs.

The entrepreneur's "running" captures several types of moral hazard problems. First, we can take the running literally: the entrepreneur is able to flee with the share $\alpha P$ without being caught, or run with the amount $P$ but with an expected fine of $(1-\alpha) P .{ }^{11}$ Second, at a reduced cost of $(1-\alpha) P<I-\tilde{n} c$ the entrepreneur can provide the consumer a product that matches the formal description but is still worthless to the consumer. ${ }^{12}$ Third, by a (possibly expected) cost $(1-\alpha) P$, the entrepreneur can convincingly claim that the project failed so that, without fearing any legal repercussions, she need not deliver the product and keep the pledges. ${ }^{13}$

Considering the entrepreneur's decision whether to run, note that she obtains a profit $P-I-c P / p$ from investing. An aggregated pledges of $P$, therefore, induces the entrepreneur to run if

$$
\alpha P>P-I-c P / p
$$

This holds not only for the extreme $\alpha=1$ but also for any $\alpha \geq 1-c / p$. In these cases, consumers rationally expect that the entrepreneur will not deliver the product so that they will not be willing to participate in the crowdfunding scheme.

\footnotetext{
${ }^{11}$ E.g. the project "Code hero" raised $\$ 170,954$ but never delivered its rewards, Polygon.com states "His critics believe he has run off with the money raised from the kickstarter campaign" (http: //www.polygon.com/2012/12/18/3781782/code-hero-kickstarter-interview, last retrieved 3 November 2015), whereas the Kickstarter campaign "Asylum Playing Cards" resulted in legal fines "against a crowdfunded project that didn't follow through on its promise to backers" (http:// wWw . atg -wa.gov/news/news-releases/ag-makes-crowdfunded-company-pay-shady-deal, last retrieved 8 November 2015).

${ }^{12}$ E.g. the crowdfunding project "Healbe GoBe" on Indiegogo raised much controversy about whether the delivered product actually works (see for instance http://blog.belgoat.com/ 24-hours-with-my-healbe-gobe/, last retrieved 8 November 2015).

${ }^{13}$ E.g. the guidelines on Kickstarter make consumers well-aware of this possibility: "If a creator is making a good faith effort to complete their project and is transparent about it, backers should do their best to be patient and understanding while demanding continued accountability from the creator." (https://www.kickstarter.com/help/faq/kickstarter\%20basics\#Acco, last retrieved 8
} November 2015). 
In the remainder of this section, we introduce two intuitive but ad hoc changes to the crowdfunding scheme $(p, T)$ that reduces entrepreneurial moral hazard. Using a mechanism design approach, the next section proves that the two changes lead to mechanisms that are indeed optimal in the class of all mechanisms.

An intuitive way to mitigate the moral hazard problem is to transfer the consumer's pledges to the entrepreneur only after having produced the good. Because the penniless entrepreneur needs at least the amount $I$ to develop the product, such a delay in payments is possible only up to the amount $I$.

Hence, a first, ad hoc step towards mitigating the moral hazard problem is to adjust the crowdfunding scheme $(p, T)$ and introduce deferred payments as follows. As before, the price $p$ represents the pledge level of an individual consumer and $T$ the target level which the sum of pledges, $P$, has to meet before the investment is triggered. Different from before however, the entrepreneur, after learning $P$, first obtains only the required amount $I$ for developing the product and receives the remaining part $P-I$ only after delivering the good to consumers.

In order to characterize crowdfunding schemes with deferred payments that prevent moral hazard, note that the entrepreneur now obtains only the payoff $\alpha I$ from a run and the payoff $P-I-c P / p$ from realizing the project. Hence, she has no incentive to run if

$$
\alpha I \leq P-I-c P / p \Rightarrow P \geq \bar{P} \equiv \frac{(1+\alpha) p I}{p-c} .
$$

In particular, the deferred crowdfunding scheme with a pledge level of $p=1$ and a target $T=(1+\alpha) I /(1-c)$ does not induce any moral hazard. Given this scheme, a consumer with value $v=1$ is willing to pledge $p=1$ and the scheme leads to an equilibrium outcome in which all consumers with $v=1$ pledge and the project is triggered when at least $T$ consumers have the willingness to pay of 1 , i.e. if $n_{1}>(1+\alpha) I /(1-c)$. Although the scheme does prevent moral hazard, it, for any $\alpha>0$, does not attain the efficient outcome, because its target level is larger than the socially efficient one; the scheme exhibits under-investment.

The information trade-off. We argued that a crowdfunding scheme with deferred payments can circumvent the moral hazard problem. Since this deferred crowdfunding scheme does not yield an efficient outcome, the question arises whether there 
are more sophisticated crowdfunding schemes that do better. To show that this is indeed the case, note first that with respect to choosing the efficient investment decision, the entrepreneur only needs to learn whether $n_{1}$ is above or below $\bar{n}$. The exact value of $n_{1}$ is immaterial.

Yet, as inequality (2) reveals, the moral hazard problem intensifies if the entrepreneur obtains full information about $P$. As discussed, this inequality has to hold for any possible realization of $P \geq T$ in order to prevent the entrepreneur from running. Because the constraint is most stringent for $P=T$, a crowdfunding scheme $(p, T)$ prevents moral hazard if and only if $T \geq \bar{P}$.

In contrast, if the entrepreneur would only learn that $P$ exceeds $T$, but not the exact value of $P$ itself, then she rationally anticipates an expected payoff

$$
E[P \mid P \geq T]-I-c E[P \mid P \geq T] / p
$$

from not running with the money. Since the conditional expectation $E[P \mid P \geq T]$ obviously exceeds $T$, a crowdfunding scheme that reveals only whether $P$ exceeds $T$ can deal with the moral hazard problem more efficiently.

Hence, in the presence of both demand uncertainty and moral hazard, the information extraction problem becomes a sophisticated one, because the extraction of demand information interacts with the moral hazard problem. As a result, one neither wants too much nor too little information revelation.

The analysis up to now has been ad hoc. By starting with a simple crowdfunding scheme that reflects all-or-nothing reward-crowdfunding schemes in practise and adapting it in two way - introducing deferred payments and reducing its informativeness - we improved its efficiency properties. The ad hoc nature of these two changes, raises the question whether even more efficient crowdfunding mechanisms exist.

In order to study this question, the next section first sets up the crowdfunding problem as one of optimal mechanism design. It subsequently formally proves that the payout-deferred and information-restricted reward crowdfunding scheme $(p, T)$ towards which we argued intuitively is indeed optimal in the class of all mechanisms. In other words, such crowdfunding schemes are an indirect implementation of the optimal direct mechanism. 


\section{Crowdfunding and Mechanism Design}

In this section we cast the entrepreneur's economic problem into a problem of mechanism design and characterize optimal mechanisms. In order to treat the entrepreneur's moral hazard, we use the framework of Myerson (1982), which handles both unobservable information and unobservable actions. This generalized framework introduces a mediator, who coordinates the communication between economic agents and gives incentive compatible recommendations concerning the unobservable actions that lead to moral hazard. One of the insights from this analysis is that crowdfunding platforms play exactly the role of a mediator in the sense of Myerson (1982). The section's main result is to confirm formally that the payout-deferred and information-restricted reward crowdfunding scheme as identified in the previous section is a (constrained) efficient mechanism.

Economic Allocations. In order to cast the entrepreneur's investment problem in a framework of mechanism design, we first make precise the feasible economic allocations: Crowdfunding seeks to implement an allocation between one cash-constrained entrepreneur, player 0 , and $n$ consumers, players 1 to $n$. It involves monetary transfers and production decisions. Concerning monetary transfers, consumers can make transfers to the entrepreneur both before and after the entrepreneur's investment decision. We denote the ex ante transfer from consumer $i$ to the entrepreneur by $t_{i}^{a}$ and the ex post transfer by $t_{i}^{p}$. Concerning the production decisions, the allocation describes whether the entrepreneur invests, $x_{0}=1$, or not, $x_{0}=0$, and whether the entrepreneur produces a good for consumer $i, x_{i}=1$, or not, $x_{i}=0$. Consequently, an economic allocation is a collection $a=(t, x)$ of transfers $t=\left(t_{1}^{a}, \ldots, t_{n}^{a}, t_{1}^{p}, \ldots, t_{n}^{p}\right) \in \mathbb{R}^{2 n}$ and outputs $x=\left(x_{0}, \ldots, x_{n}\right) \in X \equiv\{0,1\}^{n+1}$.

Feasible Allocations. By the very nature of the crowdfunding problem, the entrepreneur does not have the resources to finance the required investment $I>0$. The entrepreneur's financial constraints imply the following restrictions on feasible allocations. First, if the entrepreneur invests $\left(x_{0}=1\right)$, the transfers of the consumers must be enough to cover the investment costs $I$. Moreover, the entrepreneur can not make any net positive ex ante transfers to consumers if she does not invest $\left(x_{0}=0\right)$. Second, aggregate payments must be enough to cover the entrepreneur's investment 
and production costs. To express these two feasibility requirements, we say that an allocation $a=(t, x)$ is budget-feasible if

$$
\sum_{i=1}^{n} t_{i}^{a} \geq I x_{0} \text { and } \sum_{i=1}^{n} t_{i}^{a}+t_{i}^{p} \geq I x_{0}+c \sum_{i} x_{i} .
$$

In addition, an entrepreneur can only produce a good to a consumer if she developed it. To express this feasibility requirement, we say that an allocation $a=(t, x)$ is development feasible if, whenever the good is produced for at least one consumer, the entrepreneur invested in its development:

$$
\exists i: x_{i}=1 \Rightarrow x_{0}=1
$$

This condition logically implies that if $x_{0}=0$ then $x_{i}=0$ for all $i$.

Let the set $A \subset \mathbb{R}^{2 n} \times\{0,1\}^{n+1}$ denote the set of budget- and development-feasible allocations, i.e. allocations that satisfy (3) and (4).

Payoffs. Let the $n$-dimensional vector $v=\left(v_{1}, \ldots, v_{n}\right) \in V \equiv\{0,1\}^{n}$ represent the valuation profile of the consumers. We denote the probability of $v \in V$ by $\pi(v)$ and the conditional probability of $v_{-i} \in V_{-1} \equiv\{0,1\}^{n-1}$ given $v_{i}$ as $\pi_{i}\left(v_{-i} \mid v_{i}\right)$. Assuming that individual types are drawn independently, it holds $\pi_{i}\left(v_{-i} \mid 0\right)=\pi_{i}\left(v_{-i} \mid 1\right)$ so that we can express the conditional probability simply as $\pi_{i}\left(v_{-i}\right)$. Moreover, we assume that consumers are identical: $\pi_{i}\left(v_{-k}\right)=\pi_{j}\left(v_{-k}\right)$ for any $v_{-k} \in V_{-1}$ and $i, j$.

A feasible allocation $a \in A$ yields a consumer $i$ with value $v_{i}$ the payoff

$$
U_{i}\left(a \mid v_{i}\right)=v_{i} x_{i}-t_{i}^{a}-t_{i}^{p}
$$

and the entrepreneur the payoff

$$
\Pi(a)=\sum_{i=1}^{n}\left(t_{i}^{a}+t_{i}^{p}\right)-c \cdot \sum_{i=1}^{n} x_{i}-I x_{0} \geq 0,
$$

where the inequality follows directly from the second inequality in (3), implying that any feasible allocation yields the entrepreneur a non-negative payoff.

Efficiency. An output schedule $x \in X$ is Pareto efficient in state $v$ if and only if it maximizes the aggregate net surplus

$$
S(x \mid v) \equiv \Pi(a)+\sum_{i=1}^{n} U_{i}\left(a \mid v_{i}\right)=\sum_{i=1}^{n}\left(v_{i}-c\right) x_{i}-I x_{0} .
$$


With respect to efficiency, two different types of production decisions matter: the overall investment decision $x_{0}$ and the individual production decisions $x_{i}$. Given $v_{l}=0<c<v_{h}=1$, efficiency with respect to the individual allocations requires $x_{i}=v_{i}$. This yields a surplus of $\sum_{i} v_{i}(1-c)-I$.

Defining

$$
\bar{n} \equiv \frac{I}{1-c} ; V^{0} \equiv\left\{v: \sum_{i} v<\bar{n}\right\} ; V^{1} \equiv\left\{v: \sum_{i} v \geq \bar{n}\right\} ; \text { and } \pi^{*} \equiv \sum_{v \in V^{1}} \pi(v),
$$

we can fully characterize the Pareto efficient output schedule $x^{*}(v)$ as follows. For $v \in V^{0}$, it exhibits $x_{0}^{*}=x_{i}^{*}=0$ for all $i$. For $v \in V^{1}$, it exhibits $x_{0}^{*}=1$ and $x_{i}^{*}=v_{i}$ for all $i{ }^{14}$ Under an efficient output schedule, the entrepreneur invests only if $v \in V^{1}$, implying that $\pi^{*}$ expresses the ex ante probability that the project is executed.

Although transfers are immaterial for Pareto efficiency, we must nevertheless ensure that the efficient output schedule $x^{*}(v)$ can indeed be made part of some feasible allocation $a \in A$. In order to specify one such feasible allocation, we define the first best allocation $a^{*}(v)$ as follows. For $v \in V^{1}$, it exhibits $x_{i}=t_{i}^{a}=v_{i}=1$ and $t_{i}^{p}=0$. For $v \in V^{0}, a^{*}(v)$ is defined by $x_{i}=t_{i}^{a}=x_{i}=t_{i}^{a}=t_{i}^{p}=0$. By construction $a^{*}(v)$ is feasible and yields an ex ante expected gross surplus (gross of investment costs) of $W^{*}$, where

$$
W^{*} \equiv \sum_{v \in V^{1}} \sum_{i}^{n} \pi(v) v_{i}(1-c)
$$

We further say that an output schedule $x: V \rightarrow X$ is development efficient if

$$
x_{0}(v)=1 \Rightarrow \exists i: x_{i}(v)=1 \text {. }
$$

This condition is the converse of development feasibility (4). If it does not hold, it implies the inefficiency that there is a state $v$ in which the entrepreneur invests $I$ but no consumer consumes the good. Although technically feasible, a schedule that is not development efficient is not Pareto efficient, since it wastes the investment $I>0$.

For future reference, the following lemma summarizes these considerations.

Lemma 1 The first best allocation $a^{*}(v)$ is feasible and exhibits an output schedule that is development efficient. It yields an expected net surplus of $W^{*}-\pi^{*} I$.

\footnotetext{
${ }^{14}$ For $\sum_{i} v=\bar{n}$, the output schedule $x_{0}^{*}=x_{i}^{*}=0$ is also efficient, but this is immaterial (and can only arise for the non-generic case that $I$ is a multiple of $1-c$ ).
} 
Mechanisms. We next turn to mechanisms. A mechanism $\Gamma$ is a set of rules between the entrepreneur and the $n$ consumers that induces a game. Its outcome is an allocation $a \in A$ with payoffs $\Pi(a)$ and $U_{i}\left(a \mid v_{i}\right)$. In line with Myerson (1982), we interpret the crowdfunding platform as the mediator, who runs the mechanism; it credibly commits to enforce the rules of the game which the mechanism specifies, and it coordinates the communication between participants.

At the end of Section 3, we considered one such mechanism: the payout-deferred, information restricted, all-or-nothing reward-crowdfunding scheme $\Gamma^{C F}=(p, T)$. This mechanism induces the following game between the entrepreneur and consumers enforced by the crowdfunding platform: Each consumer $i$ sends to the platform a confidential binary message $m_{i} \in\{0,1\}$. If $\sum_{i} m_{i} p<T$, the platform enforces the allocation $t_{i}^{a}=t_{i}^{p}=x_{i}=x_{0}=0$. If $\sum_{i} m_{i} p \geq T$, the platform enforces the ex ante payments $t_{i}^{a}=I / \sum_{i} m_{i}$. In case the entrepreneur does not run, it subsequently enforces outputs $x_{i}=m_{i}$ and transfers $t_{i}^{p}=p-t_{i}^{a}$. In case the entrepreneur runs, the platform enforces outputs $x_{i}=0$ and transfers $t_{i}^{p}=0$.

The main task of this section is to demonstrate that this type of mechanism is indeed optimal. In order to do so, it is common to first introduce a different class of mechanisms: direct mechanisms.

A direct mechanism is a function $\gamma: V \rightarrow A$, which induces the following game. ${ }^{15}$ First, consumers simultaneously and independently send a (confidential) report $v_{i}^{r}$ about their values to the platform. Based on the collected reports $v^{r}$ and in line with the rules $\gamma$, the platform collects the funds $T^{a}=\sum_{i} t_{i}^{a}\left(v^{r}\right)$ from the consumers and transfers them to the entrepreneur together with the recommendation $x_{0}\left(v^{r}\right)$ about whether to invest $I$. To capture the moral hazard problem, we explicitly assume that the platform cannot coerce the entrepreneur into following the recommendation $x_{0}=$ 1. That is, the entrepreneur is free to follow or reject it. If, however, the entrepreneur follows the recommendation, the platform enforces the production schedule $x\left(v^{r}\right)=$ $\left(x_{1}\left(v^{r}\right), \ldots, x_{n}\left(v^{r}\right)\right)$ and the transfers $t_{i}^{p}\left(v^{r}\right)$. If the entrepreneur does not follow the recommendation to invest, but runs, then individual production schedules are 0 , and no ex post transfers flow, i.e. $x_{i}=t_{i}^{p}=0$. Moreover, consumers forfeit their ex ante

\footnotetext{
${ }^{15}$ Hence, we study the extreme where the entrepreneur contracts with all $n$ consumers. In section 6.2 we address extensions where the mechanism can reach only a subset of all consumers.
} 
transfers $t_{i}^{a}$.

A direct mechanism $\gamma$ is incentive compatible if its induced game as described above has a perfect Bayesian equilibrium in which 1) consumers are truthful in that they reveal their values honestly, i.e. $v_{i}^{r}=v_{i}$, and 2) the entrepreneur is obedient in that she follows the recommendation, i.e. $x_{0}=x_{0}\left(v^{r}\right)$.

To formalize the notion of truthful revelation, we define

$$
X_{i}\left(v_{i}\right) \equiv \sum_{v_{-i} \in V_{-i}} x_{i}\left(v_{i}, v_{-i}\right) \pi_{i}\left(v_{-i}\right)
$$

and

$$
T_{i}\left(v_{i}\right) \equiv \sum_{v_{-i} \in V_{-i}}\left(t_{i}^{a}\left(v_{i}, v_{-i}\right)+t_{i}^{p}\left(v_{i}, v_{-i}\right)\right) \pi_{i}\left(v_{-i}\right) .
$$

Consequently, we say that a direct mechanism $\gamma$ is truthful if

$$
v_{i} X_{i}\left(v_{i}\right)-T_{i}\left(v_{i}\right) \geq v_{i} X_{i}\left(v_{i}^{\prime}\right)-T_{i}\left(v_{i}^{\prime}\right) \text { for all } i \in I \text { and } v_{i}, v_{i}^{\prime} \in V_{i} \text {. }
$$

To formalize the notion of obedience, we define for a direct mechanism $\gamma$ the set $\mathcal{T}^{a}$ as the set of possible aggregate ex ante transfers which the mechanism can induce conditional on recommending investment:

$$
\mathcal{T}^{a} \equiv\left\{T^{a} \mid \exists v \in V: \sum_{i=1}^{n} t_{i}^{a}(v)=T^{a} \wedge x_{0}(v)=1\right\} .
$$

Given this set we define for any $T^{a} \in \mathcal{T}^{a}$ the set $V\left(T^{a}\right)$ which comprises all states that induce a recommendation to invest together with a total transfer $T^{a}$ :

$$
V\left(T^{a}\right) \equiv\left\{v \in V \mid x_{0}(v)=1 \wedge \sum_{i} t_{i}^{a}(v)=T^{a}\right\} .
$$

Upon receiving a recommendation to invest, the entrepreneur has received some transfer $T^{a} \in \mathcal{T}^{a}$ and has a belief $\pi\left(v \mid T^{a}\right)$ that the state is $v$. These beliefs are Bayes' consistent whenever

$$
\pi\left(v \mid T^{a}\right) \equiv \begin{cases}\frac{\pi(v)}{\sum_{v^{\prime} \in V\left(T^{a}\right)} \pi\left(v^{\prime}\right)} & \text { if } v \in V\left(T^{a}\right) \\ 0 & \text { otherwise. }\end{cases}
$$

With this notation, we say that a direct mechanism $\gamma$ is obedient if for any $T^{a} \in \mathcal{T}^{a}$ and after obtaining the recommendation to invest, $x_{0}=1$, the entrepreneur is better off investing than taking the money and run, given her updated belief $\pi\left(v \mid T^{a}\right)$ :

$$
\sum_{v \in V} \sum_{i=1}^{n} \pi\left(v \mid T^{a}\right)\left(t_{i}^{p}(v)-c x_{i}(v)\right)+T^{a}-I \geq \alpha T^{a}, \text { for all } T^{a} \in \mathcal{T}^{a} .
$$


We say that a direct mechanism is incentive compatible if and only if it is truthful and obedient.

By its nature, participation in the crowdfunding mechanism is voluntary so that it must yield the consumers and the entrepreneur at least their outside option. Taking these outside options as 0 , the entrepreneur's participation is not an issue, because, as argued, any feasible allocation yields the entrepreneur a non-negative payoff. In contrast, a consumer's participation in an incentive compatible direct mechanism is individual rational only if

$$
v_{i} X_{i}\left(v_{i}\right)-T_{i}\left(v_{i}\right) \geq 0 \text { for all } i \in I \text { and } v_{i} \in V_{i} \text {. }
$$

To summarize, we say that a direct mechanism $\gamma$ is feasible, if it is incentive compatible and individual rational for each consumer. ${ }^{16} \mathrm{~A}$ feasible direct mechanism yields consumer $i$ with valuation $v_{i}$ the utility

$$
U_{i}\left(v_{i}\right) \equiv v_{i} X_{i}\left(v_{i}\right)-T_{i}\left(v_{i}\right)
$$

and the entrepreneur an expected payoff

$$
\Pi=\sum_{v \in V} \pi(v) \Pi(\gamma(v))
$$

Finally, we say that two feasible direct mechanisms $\gamma=(t, x)$ and $\gamma^{\prime}=\left(t^{\prime}, x^{\prime}\right)$ are payoff-equivalent if they lead to identical payoffs to each consumer type $v_{i}$ :

$$
\sum_{v_{-i} \in V_{-i}} \pi\left(v_{-i}\right) U_{i}\left(\gamma(v), v_{i}\right)=\sum_{v_{-i} \in V_{-i}} \pi\left(v_{-i}\right) U_{i}\left(\gamma^{\prime}(v), v_{i}\right), \forall i, v_{i}
$$

and the entrepreneur:

$$
\sum_{v \in V} \pi(v) \Pi(\gamma(v))=\sum_{v \in V} \pi(v) \Pi\left(\gamma^{\prime}(v)\right)
$$

Implementability. An allocation function $f: V \rightarrow A$ specifies for any value profile $v$ an allocation $a \in A$. It is implementable if there exists a mechanism $\Gamma$

\footnotetext{
${ }^{16}$ This implicitly assumes that the mechanism has "perfect consumer reach" in that every consumer is aware and can participate in the mechanism. As an extension that yields important additional insights, Subsection 6.2 studies the effect of imperfect consumer reach.
} 
such that the induced game has a perfect Bayesian equilibrium outcome in which the induced allocation coincides with $f(v)$ for every $v \in V$. In this case, we say $\Gamma$ implements $f$.

Likewise, an output schedule $x: V \rightarrow X$ specifies for any value profile $v$ an output schedule $x \in X$. It is implementable if there exists a mechanism $\Gamma$ such that the induced game has a perfect Bayesian equilibrium outcome in which the induced output coincides with $x(v)$ for every $v \in V$. In this case, we say $\Gamma$ implements output schedule $x(\cdot)$.

By the revelation principle, an allocation function $f(\cdot)$ is implementable if and only if there exists a feasible direct mechanism $\gamma$ with $\gamma(v)=f(v)$ for any $v \in V$. Likewise, an output schedule $x(\cdot)$ is implementable if and only if there exists a direct mechanism $\gamma=\left(x_{\gamma}, t_{\gamma}\right)$ such that $x_{\gamma}(v)=x(v)$ for any $v \in V$. Hence, as usual, the revelation principle motivates incentive compatibility as one of the defining requirements of feasibility. A first question that arises is whether an efficient output schedule is always implementable. Considering a specific version of the model, the next proposition demonstrates that this is not the case:

Proposition 1 For $I=n-1 / 2, \alpha=1$, and $c=0$, the efficient output schedule $x^{*}(v)$ is not implementable.

The proposition implies that, in general, the efficient output is not implementable. The main driver behind this inefficiency result is a tension between the entrepreneur's budget constraint and the moral hazard problem. For consumers to make sure that the entrepreneur realizes her project, it does not suffice to give her simply the required amount $I$ to invest. Due to the moral hazard problem, she must also be given an incentive to actually invest this money. The proposition shows that for the efficient output schedule $x^{*}$ this is, in general, not possible.

The proposition raises questions about which output schedules are generally implementable and about the conditions under which the efficient schedule is implementable. To answer these questions we investigate the mechanism design problem further. The following lemma shows that with respect to development-efficient allocations, we may reduce the class of feasible direct mechanisms further. 
Lemma 2 If $\gamma=(t, x)$ is feasible and $x$ is development-efficient then there is a feasible and payoff equivalent direct mechanism $\hat{\gamma}=(\hat{t}, x)$ with

$$
\sum_{i} \hat{t}_{i}^{a}(v)=I x_{0}(v), \forall v \in V
$$

The lemma implies that with respect to development-efficient mechanisms there is no loss of generality in restricting attention to feasible direct mechanisms that give the entrepreneur exactly the amount $I$ if the entrepreneur is to develop the product. The lemma therefore makes precise the suggestion of the previous section that a mechanism should provide the entrepreneur with the minimal amount of information for reducing demand uncertainty; effectively, she should only be told that the demand of consumers ensures that the project has a positive NPV, but not more. The main step in proving this result is to show that obedience remains satisfied when we replace different aggregate levels of ex ante payments by a single one. ${ }^{17}$

The lemma simplifies the mechanism design problem in two respects. First, under condition (12), condition (3) reduces to

$$
\sum_{i=1}^{n} t_{i}^{p}(v) \geq c \sum_{i} x_{i}(v)
$$

Second, under condition (12), we have $\mathcal{T}^{a}=\{I\}$ so that the obedience constraint (8) must only be respected with regard to $I$ :

$$
\sum_{v \in V} \sum_{i=1}^{n} \pi(v \mid I)\left(t_{i}^{p}(v)-c x_{i}(v)\right) \geq \alpha I
$$

\section{5 (Constrained) efficient mechanisms}

In this section we characterize second best mechanisms $\gamma^{s b}=\left(x^{s b}, t^{s b}\right)$ that maximize aggregate surplus in the presence of demand uncertainty and moral hazard. We are especially interested in determining the circumstances under which these second best mechanisms do not implement the efficient output schedule $x^{*}$.

Recall that a feasible direct mechanism $\gamma$ yields a surplus of

$$
\sum_{v \in V} \pi(v) S(x(v) \mid v)=\sum_{v \in V} \pi(v)\left[\sum_{i}^{n}\left(v_{i}-c\right) x_{i}(v)-I x_{0}(v)\right] .
$$

\footnotetext{
${ }^{17}$ The lemma fails for development-inefficient mechanisms so that we cannot dispense with the restriction to development-efficient mechanisms.
} 
Clearly $\gamma^{s b}$ cannot yield more than $W^{*}-\pi^{*} I$, which is generated under the efficient output schedule $x^{*}$. Indeed, Proposition 1 showed that, in general, $\gamma^{\text {sb }}$ achieves strictly less.

As $\gamma^{s b}$ is necessarily development-efficient, we can find it by maximizing (15) subject to the constraints (7), (9), (12), (13), and (14), because these constraints characterize the set of implementable allocation functions that are development-efficient. The maximization problem yields the following partial characterization of $\gamma^{s b}$ :

Lemma 3 The individual rationality constraint of consumers with the high value $v_{i}=1$ does not restrict the second best mechanism $\gamma^{s b}$. The second best mechanism exhibits $x_{i}\left(0, v_{-i}\right)=X_{i}(0)=T_{i}(0)=0$, and $T_{i}(1)=X_{i}(1)$ for all $i=1, \ldots, n$.

It follows from the previous lemma that the second best mechanism $\gamma^{s b}$ is a solution to the problem

$$
\begin{aligned}
\mathcal{P}: \max _{x(.), t(.)} & \sum_{v \in V} \pi(v)\left[\sum_{i}^{n}\left(v_{i}-c\right) x_{i}(v)-I x_{0}(v)\right] \\
\text { s.t. } & T_{i}(1)=X_{i}(1) \text { for all } i \\
& \sum_{v \in V} \sum_{i=1}^{n} \pi(v \mid I)\left(t_{i}^{p}(v)-c x_{i}(v)\right) \geq \alpha I \\
& T_{i}(0)=0 \text { for all } i \\
& \sum_{i=1}^{n} t_{i}^{a}(v)=I x_{0}(v) ; \\
& \sum_{i=1}^{n} t_{i}^{p}(v) \geq \sum_{i} c x_{i}(v) \\
& x_{i}(v)=1 \Rightarrow x_{0}(v)=1 \\
& x_{i}\left(0, v_{-i}\right)=0, \forall v_{-i} \in V_{-i} .
\end{aligned}
$$

Recalling that $\pi^{*}$ represents the ex ante probability that the project is executed under the efficient schedule $x^{*}$, we obtain the following result.

Proposition 2 The efficient output schedule $x^{*}$ is implementable if and only if $W^{*} \geq$ $\bar{W}^{\alpha} \equiv(1+\alpha) \pi^{*} I$. If implementable, the indirect payout-deferred, informationrestricted, all-or-nothing reward-crowdfunding scheme $\bar{\Gamma}^{C F}=(\bar{p}, \bar{T})$ with $\bar{p} \equiv 1$ and $\bar{T} \equiv I /(1-c)$ implements $x^{*}$. 
Proposition 2 makes precise the parameter constellation under which the first best $x^{*}$ is implementable: only if the efficient production schedule $x^{*}$ generates a surplus that exceeds the ex ante expected investment costs $(1+\alpha)$ times. For values of $W^{*}$ in between $\pi^{*} I$ and $(1+\alpha) \pi^{*} I$, the optimal mechanism exhibit inefficiencies.

Intuitively, the driver behind these inefficiencies is a tension between the entrepreneur's budget constraint and her moral hazard problem. For consumers to make sure that the entrepreneur realizes her project, it does not suffice to give her simply the required amount $I$ to invest. Due to the moral hazard problem, she must also be given an incentive to actually invest this money. As the proposition shows, this effectively requires consumers to pay the entrepreneur the run-away payoff $\alpha I$. The project is therefore only worthwhile if the project's revenue recovers the augmented investment cost $(1+\alpha) I$.

Effectively, the proposition shows that the combination of the entrepreneur's budget constraint and her moral hazard problem increases investment costs by a factor $\alpha$. It prevents first best outcomes if the expected gross surplus $W^{*}$ is too small.

Whenever the ex ante gross surplus does not exceed the expected investment costs by the factor $\alpha$, the efficient output schedule, $x^{*}$, is not implementable so that the second best output schedule $x^{s b}$ does not coincide with $x^{*}$. We next characterize both the second best and the type of inefficiencies it exhibits.

Proposition 3 For $W^{*}<\bar{W}^{\alpha}$, the constrained efficient output schedule $x^{\text {sb }}$ exhibits i) $x_{i}^{s b}(v)=v_{i}$ whenever $x_{0}^{s b}(v)=1$; ii) $x_{0}^{s b}(v)=0$ whenever $x_{0}^{*}(v)=0$; and iii) $x_{0}^{s b}(v)=1$ whenever $\sum v_{i}>(1+\alpha) I /(1-c)$. Moreover, an indirect payout-deferred, information restricted, all-or-nothing reward-crowdfunding scheme $\Gamma^{C F}=(p, T)$ implements $x^{s b}$.

The first part of the proposition shows that the constrained efficient output schedules are only distorted with respect to the investment decision but not to the individual assignments. The second part of the proposition shows that the second best output schedule is distorted downwards rather than upwards. The third part shows that at most the allocations for which aggregate valuations lie in the range between $W^{*}$ and $\bar{W}^{\alpha}$ are downward distorted. This final statement also implies that for the 
constrained efficient output schedule it matters only whether the sum of valuations exceed a target level $T$. As a result, the second best scheme can be implemented indirectly by a crowdfunding scheme $(1, T)$.

\section{Interpretation and Discussion}

This section interprets the optimal direct mechanism as derived in the previous section and relates it to crowdfunding platforms in practise. It further discusses extensions and robustness of the results.

\subsection{Comparison to current crowdfunding platforms}

Relating our theoretical results to current crowdfunding platforms, our first observation concerns the role of the crowdfunding platform itself. In our formal analysis the platforms structures the communication between entrepreneur and consumers, and executes the mechanism. We note that this is fully in line with the role that crowdfunding platforms play in practise. Platforms such as Kickstarter emphasize that they are not involved in the development of the projects themselves and take no responsibility in guaranteeing the entrepreneur's project. ${ }^{18}$ Wikipedia therefore calls the platform "a moderating organization". Tellingly, the technical term of the platform's role in (extended) mechanism design is "mediator" (e.g. Myerson 1982). Although the platform's role seems only minor, it is nevertheless crucial. Due to commitment problems, it cannot be performed by either the entrepreneur or the consumers.

A second notable feature of optimal direct mechanisms is that they explicitly condition the entrepreneur's investment decision on the sum of reported valuations rather than each consumer's report individually. This is consistent with the many "all-or-nothing" pledge schemes of popular reward crowdfunding platforms such as Kickstarter. In other words, these schemes are indirect mechanisms that implement the conditional investment.

\footnotetext{
${ }^{18}$ See https://en.wikipedia.org/wiki/Crowdfunding and https://www.kickstarter.com/ help/faq/kickstarter\%20basics\#Acco for explaining the limited involvement of Kickstarter, last retrieved 8 November 2015.
} 
A third feature of optimal direct mechanisms is that they do not exhibit negative transfers. Hence, at no point in time the entrepreneur needs to pay consumers any money. In particular, she does not share her revenue after the investment: $t_{i}^{p}(v) \geq$ 0. Consequently, optimal mechanisms do not turn consumers into investors; the optimal crowdfunding scheme is not investment-based. This feature is consistent with current reward crowdfunding: a crowdfunding consumer receives only a nonmonetary reward for his pledged contribution. Reward-crowdfunding schemes such as Kickstarter explicitly prohibit any monetary transfers to crowdfunders. ${ }^{19,20}$

A fourth feature of optimal direct mechanisms is a deferred payout to prevent moral hazard. Some but definitely not all crowdfunding platforms do so. For instance, PledgeMusic, a crowdfunding platform specialized in raising money for music recordings, uses deferred payouts to prevent fraud. ${ }^{21}$

A final notable feature of optimal direct mechanisms is that they provide only information about whether the sum of pledges exceeds the target and not the total sum of pledges itself. In line with Lemma 2 any additional information is not needed to implement (constrained) efficient outcomes, and schemes that provide more information may exacerbate the moral hazard problem. Current crowdfunding platforms do not reflect this feature. Currently all crowdfunding platforms are fully transparent and announce publicly the total amount of pledges rather than just whether the target level was reached.

To summarize, current all-or-nothing reward crowdfunding platforms reflect many crucial features of the optimal mechanisms underlying our theoretical model. They, however, do not seem to deal with moral hazard optimally. The next section addresses especially this latter point.

\subsection{Limited Consumer Reach}

The previous section suggests that current crowdfunding schemes deal with moral hazard suboptimally. In particular, they rarely use deferred payments, which, as shown by PledgeMusic, seems relatively straightforward to implement. On the other

\footnotetext{
${ }^{19}$ See https://www.kickstarter.com/rules?ref=footer, last retrieved 22 July 2015.

${ }^{20}$ The next section argues however that a limited reach of the platform results in crowd-investment.

${ }^{21}$ See http: //www . pledgemusic.com/blog/220-preventing-fraud, last retrieved 20 July 2015.
} 
hand, there seems nevertheless little indication that in practise fraud is a prevalent problem in crowdfunding. Mollick (2013), for instance, reports that fraudulent failures for Kickstarter projects are very rare (well below 5\%).

Since crowdfunding platforms currently reach only a very small part of potential demand, this section argues that even though crowdfunding schemes do not use deferred payout explicitly, they do so implicitly. The argument is that the entrepreneur's prospect to sell her products to consumers who did not participate in crowdfunding acts as a direct substitute for deferred payments. ${ }^{22}$

Motivated by the observation that crowdfunding allows entrepreneurs to contract with consumers before their investment, our formal analysis took this idea to the extreme and implicitly assumed that the entrepreneur could contract with every potential consumer. Given this extreme position, the revelation principle implies that there is indeed no loss of generality in assuming that mechanisms allow consumers to acquire the product only through the mechanism. This evidently changes when, for some exogenous reason, not all consumers can participate in the mechanism. In practise this is a highly relevant concern, because a share of consumers may fail to notice the crowdfunding scheme, not have access to the internet, or only arrive in the market after the product has been developed. Hence, a relevant extension of our framework is to consider mechanisms, which, for some exogenous reason, have an imperfect consumer-reach.

In order to make this more concrete, consider an extension of the model in which only a share of $\beta \in(0,1)$ can partake in the mechanism. Already the pure proportional case that a consumer's ability to participate is independent of his valuation, yields new important insights.

Note first that for this pure proportional case, the crowdfunding scheme is still able to elicit the project's value: a pledge by $\tilde{n}$ consumers means that the project is worth $n_{1}=\tilde{n} / \beta$. Consequently, investment is efficient if and only if

$$
\tilde{n} / \beta \geq I /(1-c) \Rightarrow \tilde{n} \geq \bar{n}(\beta) \equiv \beta I /(1-c)
$$

It is straightforward to see that the previous analysis still applies when we factor in $\beta$.

\footnotetext{
${ }^{22}$ Interestingly, this may not be the case for very specialized crowdfunding platforms such as PledgeMusic, who do use explicit deferred payout systems.
} 
In particular, the efficient output scheme is implementable for $W^{*} \geq(1+\alpha) \pi^{*} I \beta{ }^{23}$

In order to see that, with limited consumer reach, a classic reward crowdfunding scheme $(p, T)$ without deferred payments can withstand moral hazard even for the extreme case $\alpha=1$, note that when only a share of $\beta$ potential consumers can be reached, inequality (1), which describes the condition under which the entrepreneur has a strict incentive to run, changes to

$$
\alpha P>P / \beta-I-c P /(p \beta) \Rightarrow \beta>\bar{\beta} \equiv \frac{1-c / p}{\alpha+I / P} .
$$

Hence, whereas, under full consumer reach $(\beta=1)$, a reward crowdfunding scheme $(p, T)$ without deferred payments is unable to withstand moral hazard whenever $\alpha>1-c / p-I / P$, it does withstand moral hazard when its consumer reach is limited to $\beta<\bar{\beta}$. The reason for this follows the logic behind deferred payments: the limited consumer reach effectively implies that a pledge level $P$ constitutes a deferred payment of $P / \beta-P>0$.

Apart from reducing the threat of moral hazard, the extension reveals an important additional economic effect: consumers may become actual investors when the share of crowdfunding consumers $\beta$ is small. To see this, note that, because the entrepreneur needs the amount $I$ to develop the product, the (average) ex ante transfer of a pledging consumer needs to be at least $I / \tilde{n}$. When $\beta$ is small in the sense that $\bar{n}(\beta)$ is smaller than 1 , it follows that for $\tilde{n}$ close to $\bar{n}(\beta)$, the consumer's ex ante transfer exceeds his willingness to pay. Individual rationality then implies that the ex post transfer to the consumer is negative. Hence, the optimal mechanism turns consumers in investors; they finance the entrepreneur's investment and share in her revenues.

As noted, reward crowdfunding schemes such as Kickstarter explicitly prohibit monetary transfers to crowdfunders. Our formal analysis confirms that this is indeed not needed if the investment $I$ is small compared to the number of crowdfunding consumers, but for large investments such restrictions may matter. ${ }^{24}$

\footnotetext{
${ }^{23}$ This "proportionality" property holds because the derived efficient scheme extracts all rents from consumers and the entrepreneur can implement the efficient outcome by using the scheme as derived and set a price $p=1$ to the $(1-\beta) n$ consumers who can only participate after the good has been developed.

${ }^{24}$ Ordanini et al. (2011) report the case of Cameesa, a Chicago based clothing company
} 
Finally note that all-or-nothing crowdfunding schemes also give consumers a strict incentive to participate in the crowdfunding scheme, even if they have the option to wait and buy the product later in the after market. This is so, because a consumer may be pivotal for the entrepreneur's decision whether to produce the good. Hence, facing a crowdfunding scheme $(p, T)$ a consumer is strictly better off participating (provided that he expects that the after market price is not lowered, which in our model would indeed not be the case). Hence, next to eliciting the consumer's valuation in an incentive compatible manner, crowdfunding schemes also exhibit features, which support the incentive compatibility of participation. ${ }^{25}$

\subsection{Entrepreneur's private cost information}

Both the first best and the optimal (constrained) efficient mechanism condition on the entrepreneur's investment cost $I$ and marginal cost $c$. The optimal mechanism, therefore, exploits the assumption that costs are public information. In practise, one may worry that the entrepreneur is better informed about these costs than consumers. In this case, the costs become the entrepreneur's private information so that if the mechanism is to condition on this information, it must incentivize the entrepreneur to reveal it. This may potentially lead to new distortions.

Note that because the optimal mechanism implements an allocation in which the entrepreneur obtains all the rents, one might be tempted to argue that the entrepreneur's incentives are fully aligned with social welfare so that the mechanism automatically gives her the proper incentives to reveal her private information truthfully. This reasoning is however incomplete, because it only considers a unilateral deviation. Hence, even though the reasoning implies that a single deviation to misreport costs is not profitable, the combined deviation of misreporting costs and, which in 2008 introduced an "all-or-nothing" crowdfunding model, but also shared revenue with its crowdfunders. Supporters of a successful project not only obtained the shirt, but also shared in some of the revenue of its future sales. (see http://www.cnet.com/news/ cameesa-a-threadless-where-customers-are-also-investors/, last retrieved 22 July 2015).

${ }^{25}$ Next to the probability to be pivotal and the consumer's expectation of the price in the after market, a consumer's specific incentives to participate will also depend on other factors from which our model abstracts: time-preferences, the probability that the project will succeed, and the possibility that the consumer can better judge the product after it has been successfully produced. 
subsequently, running away with the money run may be profitable. Therefore, the crowdfunding scheme $\bar{\Gamma}^{C F}$ implements the first best with private information about costs only if $W^{*} \geq \bar{W}^{\alpha}$ and a combined deviation is not profitable.

To examine the implications of this in closer detail, suppose that $W^{*} \geq \bar{W}^{\alpha}$ so that, under the assumption that $I$ and $c$ are public information, the efficient output $x^{*}$ is implementable by the scheme $\bar{\Gamma}^{C F}$. Now first assume that only $c$ is private information. It is then straightforward to show that, the scheme $\bar{\Gamma}^{C F}$ automatically induces the entrepreneur to report $c$ truthfully, provided that she does not run away. In order to see that $\bar{\Gamma}^{C F}$ does not imply an incentive compatibility with respect to the combined deviation of misreporting and running away, define $\bar{\pi}(T)$ as the probability that the sum of consumer valuations exceeds $T$, i.e.

$$
\bar{\pi}(T) \equiv \sum_{\left\{v: \sum_{i} v_{i} \geq T\right\}} \pi(v) .
$$

Now suppose the platform offers the scheme $\bar{\Gamma}^{C F}$ with the target $\bar{T}=I /(1-c)$, but, not observing $c$, asks the entrepreneur to report it before implementing the scheme. Instead of reporting truthfully and obtain the gross revenue $W^{*}$, the strategy to report $c^{r}$ and subsequently run away yields the entrepreneur $\alpha \bar{\pi}\left(I /\left(1-c^{r}\right)\right) I$. Hence, the best deviation for the entrepreneur is to report $c^{r}=0$ and subsequently run away with the payment $I$, yielding the deviation payoff $\alpha \bar{\pi}(I) I$. With private information about the cost $c$, the scheme $\bar{\Gamma}^{C F}$ therefore withstands moral hazard only if $W^{*}-\pi^{*} I \geq \alpha \bar{\pi}(I) I$, whereas, with public information about the cost $c$, it withstands moral hazard for $W^{*}-\pi^{*} I \geq \alpha \pi^{*} I$. Since $\pi^{*}=\bar{\pi}(I /(1-c)) \leq \bar{\pi}(I)$, the moral hazard constraint when $c$ is private information is stricter. This reasoning leads to the following lemma.

Lemma 4 Suppose I is public information, whereas $c$ is the entrepreneur's private information. Then the crowdfunding contact $\bar{\Gamma}^{C F}$ implements the efficient output schedule $x^{*}$ if and only if $W^{*} \geq\left(\pi^{*}+\alpha \bar{\pi}(I)\right) I$.

A comparison of the lemma to Proposition 2 reveals that with private information about $c$, the efficient output is more difficult to implement when $c$ is private information. The intuition for this result is that by reporting a lower cost $c$, the entrepreneur can raise the probability that the project is financed and this increases the threat of moral hazard. 
It is straightforward to extend the previous reasoning to the case when also $I$ is private information. In this case, an implementation of $\bar{\Gamma}^{C F}$ requires the entrepreneur to report both the investment cost $I$ and the marginal costs $c$. Again, misreporting $(I, c)$ does not lead to a higher payoff if the entrepreneur does not run, while reporting $\left(I^{r}, c^{r}\right)$ and subsequently running away yields the pay $\bar{\pi}\left(I^{r}\right) I^{r}$. Defining

$$
\hat{I}=\arg \max _{I^{r}} \bar{\pi}\left(I^{r}\right) I^{r}
$$

the maximum deviation payoff is $\alpha \bar{\pi}(\hat{I}) \hat{I}$. Hence, the scheme $\bar{\Gamma}^{C F}$ withstands moral hazard if $W^{*}-\pi^{*} I \geq \alpha \bar{\pi}(\hat{I}) \hat{I}$. The reasoning leads to the following proposition.

Proposition 4 Suppose $(I, c)$ are the entrepreneur's private information. Then the crowdfunding contact $\bar{\Gamma}^{C F}$ implements the efficient output schedule $x^{*}$ if and only if $W^{*} \geq \pi^{*} I+\alpha \bar{\pi}(\hat{I}) \hat{I}$.

The proposition shows that the entrepreneur's private information about costs does not affect our main qualitative results. In particular, both the result that efficiency is implementable when the ex ante gross surplus $W^{*}$ is large enough and the result that moral hazard effectively implies a mark-up on the investment costs still hold. Note also that if there is no moral hazard $(\alpha=0)$, then private information about costs does not affect the optimal crowdfunding mechanism at all. Hence, private information about costs only affect outcomes to the extent that it intensifies the moral hazard problem proportionally.

\subsection{Crowdfunding and price discrimination}

In our formal analysis, we assumed that consumers either do not value the good $\left(v=v_{l}=0\right)$ or value it at the same positive amount $\left(v=v_{h}=1\right)$. We stress that this assumption should not be understood as a simplifying one, but as one that, by contrasting it to the existing literature, allows us to identify different strengths and weaknesses of crowdfunding schemes.

To make this more precise, note that a defining features of a crowdfunding scheme $(p, T)$ is that it conditions the investment decision on the sum of pledges. Cornelli (1996, p.18), however, explicitly shows that, for achieving optimal price discrimination, the actual composition of this sum rather than the sum itself matters. As 
a result, crowdfunding schemes cannot deal with the price-discrimination problem optimally (see also Barbieri and Malueg 2010). In contrast, our results show that, even in the presence of entrepreneurial moral hazard, conditioning on the sum of pledges is optimal when eliciting the overall value of the project does not require price discrimination.

Hence, by restriction to our binary consumer valuations and comparing our results to the earlier literature, we are able to identify two different economic effects of crowdfunding and compare the ability of crowdfunding to take advantage of them: whereas crowdfunding schemes are optimal tools for "project-value screening", where the aggregate valuation of consumers matters, they are suboptimal tools for "consumerspecific-screening", which is a defining feature of price discrimination.

The distinction between the two modes of screening is also important for evaluating the welfare effects of crowdfunding: project-value screening unambiguously benefits welfare and consumers, whereas the welfare properties of consumer-specificscreening as a tool for price discrimination are, as is well-known, ambiguous.

In practise however the two screening modes are tightly connected and it is therefore hard to isolate them empirically. Indeed, anecdotal evidence suggests that the current crowdfunding schemes are also used for price-discrimination. ${ }^{26}$ Moreover, current crowdfunding platforms offer extensive evaluation tools that enable entrepreneurs to obtain much more information than just whether there is enough demand for covering the costs of the project. We however point out that if the main role of the schemes is to provide a tool of price-discrimination, then one should expect schemes which do not condition the project on the sum of pledges.

\subsection{Crowdfunding vs. Venture Capitalists}

By enabling direct interactions with consumers before the investment, crowdfunding leads to a transformation of the entrepreneurial business model. Ordanini et al. (2011) emphasize that the transformation takes place at a fundamental level, blur-

\footnotetext{
${ }^{26}$ E.g. The Economist (2010) reports the concrete example of a book publisher planning to fund a renewed publication of a sold-out book: "his efforts to tease out lenders' price sensitivity from previous Kickstarter projects showed that a $\$ 50$ contribution was the most popular amount. It also proved the largest dollar component for the highest-grossing Kickstarter projects."
} 


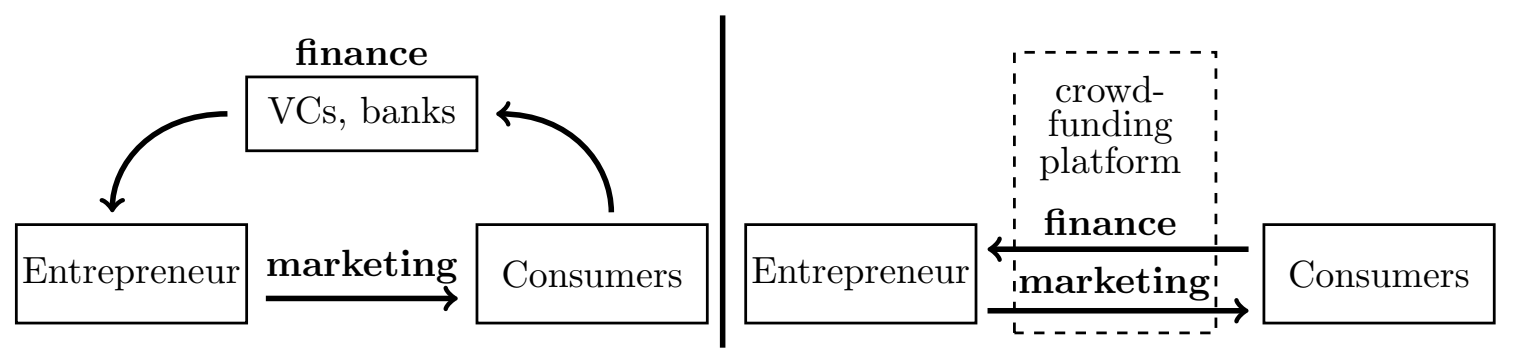

Figure 1: Traditional entrepreneurial financing (left) vs. reward crowdfunding (right)

ring the traditional separation of finance and marketing. ${ }^{27}$ Figure 1 illustrates this transformation. In the traditional model, venture capitalists (or banks) attract capital from consumers to finance entrepreneurs, who subsequently use this capital to produce goods and market them to consumers. In this traditional model, finance and marketing are naturally separated and run along different channels. In contrast under reward crowdfunding, finance and marketing run along the same channel: the crowdfunding platform.

Although this fundamental perspective is correct if one views reward crowdfunding as an exclusive alternative to specialized venture capitalists, we emphasize that crowdfunding and venture capital financing are not mutually exclusive. On the contrary, we view the two forms as highly complementary. In line with Diamond (1984), we see the advantage of venture capitalists (or banks) in reducing the moral hazard problem, which in terms of the paper's model implies a reduction in $\alpha$. In contrast, the strength of crowdfunding lies in learning about the economic value of the project.

Because the analysis of a fully-fledged model which combines venture capitalists and crowdfunding lies outside the scope of the current paper, we just mention that we see no reason why a venture capitalist may not use crowdfunding to learn about demand or why after a successful crowdfunding campaign an entrepreneur may not approach a venture capitalist. Indeed, Dingman (2013) reports that exactly this occurred in the case of the Pebble Smart Watch. Venture capitalist decided to support the entrepreneur's project only after a successful crowdfunding campaign

\footnotetext{
${ }^{27}$ In contrast, "investment-based crowdfunding" upholds the traditional separation between finance and marketing, because the consumers and the crowd-investors are typically not the same economic agents.
} 
on Kickstarter. Quoting a managing partner of a venture capitalist firm: "What venture capital always wants is to get validation, and with Kickstarter, he [i.e. the entrepreneur] could prove there was a market."

\section{Conclusion}

Crowdfunding provides the innovation that, already before the product is developed, an entrepreneur can write contracts with her potential consumers. In the presence of demand uncertainty, this enables entrepreneurs to use crowdfunding as a tool that screens for valuable projects. Our formal analysis confirms that optimal mechanisms do take on this particular role of screening. Current all-or-nothing reward crowdfunding schemes such as Kickstarter reflect the main features of these optimal mechanisms. In particular, they are consistent with the idea that these schemes are used to improve the selection of entrepreneurial projects. This promotes social welfare.

Our analysis further shows that, despite the effectiveness of reward crowdfunding schemes in screening for project value, their susceptibility to entrepreneurial moral hazard may prevent the implementation of fully efficient outcomes. In the presence of moral hazard, crowdfunding can attain fully efficient outcomes only if the project's ex ante expected gross return exceeds its ex ante expected investment costs by a markup whose size reflects the severeness of the moral hazard problem. Constrained efficient mechanisms exhibit underinvestment, but still reflect crucial features of current allor-nothing reward crowdfunding schemes.

Because crowdfunding schemes by themselves are, in the presence of moral hazard, unable to attain efficiency in general, we see them as complements rather than substitutes for traditional venture capital. We therefore expect a convergence of the two financing forms. Venture capitalists provide their economic benefit in reducing moral hazard. Crowdfunding platforms provide their economic benefit in learning about the project's value. Current policy measures such as the US JOBS Act and its implementation in SEC (2015) will make such mixed forms of crowdfunding and more traditional venture capitalism easier to develop and take advantage of their respective strengths. The website of the crowdfunding platform Rockethub already 
explicitly mentions this possible effect of the JOBS Act. ${ }^{28}$

Finally, in order to focus on the trade-off between demand uncertainty and entrepreneurial moral hazard - which we view as two fundamental first order effects in crowdfunding - our analysis necessarily abstracts from many other relevant aspects of crowdfunding. For instance, we do not address the role of crowdfunders in promoting the product, the dynamics in pledging behavior in actual crowdfunding schemes, or consumer uncertainty about the quality of the product unrelated to moral hazard. Apart from pointing out that crowdfunding and external capital provision in the form of venture capital are complements, we also do not provide a formal analysis of the interaction between external financing and reward crowdfunding. We moreover do not address possible issues concerning the platform's commitment to enforce the mechanism honestly. Since the platform is a long-term player we conjecture that its honesty can be uphold by well-known reputational arguments in repeated games (see Strausz, 2005). This however requires payments to the platform, another aspect we do not touch upon. In addition, we consider entrepreneurs, who produce a pure private good without any network effects or other externalities between consumers. Even though we consider all these issues important and relevant, they lie outside the scope of the current investigation.

${ }^{28}$ See http://www.rockethub.com/education/faq\#jobs-act-index, last retrieved 22 July 2015. 


\section{Appendix}

This appendix collects the formal proofs.

Proof of Lemma 1: Follows directly from the text

Q.E.D.

Proof of Proposition 1: Let $\mathbf{1}^{n}$ denote the vector $(1, \ldots, 1) \in \mathbb{R}^{n}$. Since $\bar{n}=$ $I /(1-c)=n-1 / 2$, it follows $V^{1}=\left\{1^{n}\right\}$ and $V^{0}=V \backslash V^{1}$ so that the efficient output schedule $x^{*}(v)$ exhibits $x_{0}^{*}(v)=x_{i}^{*}(v)=0$ for $v \neq \mathbf{1}^{n}$, and $x_{0}^{*}(v)=x_{i}^{*}(v)=1$ for $v=\mathbf{1}^{n}$. We show, by contradiction, that a feasible direct mechanism $\gamma^{*}$ that implements $x^{*}(v)$ does not exist.

For suppose to the contrary that such a direct mechanism does exist, then there exists a transfer schedule $t$ so that the direct mechanism $\gamma^{*}=\left(x^{*}, t\right)$ is feasible. Since $x_{0}^{*}(v)=1$ implies $v=1^{n}$, it follows that $\mathcal{T}^{a}$ is a singleton and for all $T^{a} \in \mathcal{T}^{a}$, it holds $V\left(T^{a}\right)=\left\{1^{n}\right\}$. Consequently, $p\left(1^{n} \mid T^{a}\right)=1$ and $\pi\left(v \mid T^{a}\right)=0$ for all $v \neq 1^{n}$. Since $\alpha=1$ and $c=0,(8)$ rewrites after multiplying by $p\left(\mathbf{1}^{n}\right)$ therefore as

$$
\sum_{i=1}^{n} t_{i}^{p}\left(\mathbf{1}^{n}\right) p\left(\mathbf{1}^{n}\right) \geq \operatorname{Ip}\left(\mathbf{1}^{n}\right) .
$$

Since $x_{0}\left(1^{n}\right)=1$ the first inequality in (3) implies after multiplying with $p\left(\mathbf{1}^{n}\right)$

$$
\sum_{i=1}^{n} t_{i}^{a}\left(\mathbf{1}^{n}\right) p\left(\mathbf{1}^{n}\right) \geq \operatorname{Ip}\left(\mathbf{1}^{n}\right) .
$$

Note further that the second inequality in (3) for each $v \neq \mathbf{1}^{n}$ implies

$$
\sum_{i=1}^{n} t_{i}^{a}(v)+t_{i}^{p}(v) \geq 0
$$

Multiplying with $\pi(v)$ and adding over all $v \neq \mathbf{1}^{n}$ yields

$$
\sum_{v \neq \mathbf{1}^{n}} \sum_{i=1}^{n}\left(t_{i}^{a}(v)+t_{i}^{p}(v)\right) \pi(v) \geq 0
$$

Combining (24), (25), and (27) yields

$$
\sum_{i} \sum_{v \in V}\left(t_{i}^{a}(v)+t_{i}^{p}(v)\right) \pi(v) \geq 2 \operatorname{Ip}\left(\mathbf{1}^{n}\right)=(2 n-1) p\left(\mathbf{1}^{n}\right)
$$

where the equality uses $I=n-1 / 2$.

We now show that (28) contradicts (9). First note that (9) for $v_{i}=0$ implies after a multiplication by $p_{i}(0)$ for each $i$

$$
\sum_{v_{-i} \in V_{-i}}\left(t_{i}^{a}\left(0, v_{-i}\right)+t_{i}^{p}\left(0, v_{-i}\right)\right) p\left(0, v_{-i}\right) \leq 0
$$


Summing over $i$ it follows

$$
\sum_{i} \sum_{v_{-i} \in V_{-i}}\left(t_{i}^{a}\left(0, v_{-i}\right)+t_{i}^{p}\left(0, v_{-i}\right)\right) p\left(0, v_{-i}\right) \leq 0
$$

Likewise, since $X_{i}(1)=p_{i}\left(\mathbf{1}^{n-1}\right)$, (9) for $v_{i}=1$ implies after a multiplication with $p_{i}(1)$ and using $p_{i}(1)=p\left(1, v_{-i}\right)$ that for each $i$

$$
\sum_{v_{-i} \in V_{-i}}\left(t_{i}^{a}\left(1, v_{-i}\right)+t_{i}^{p}\left(1, v_{-i}\right)\right) p\left(1, v_{-i}\right) \leq p\left(\mathbf{1}^{n}\right)
$$

Summing over $i$ yields

$$
\sum_{i} \sum_{v_{-i} \in V_{-i}}\left(t_{i}^{a}\left(1, v_{-i}\right)+t_{i}^{p}\left(1, v_{-i}\right)\right) p\left(1, v_{-i}\right) \leq p\left(\mathbf{1}^{n}\right) n
$$

Combining (30) and (32) yields

$$
\sum_{i} \sum_{v \in V}\left(t_{i}^{a}(v)+t_{i}^{p}(v)\right) \pi(v) \leq p\left(\mathbf{1}^{n}\right) n
$$

But since $2 n-1>n$, this contradicts (28).

Q.E.D.

Proof of Lemma 2: Fix a feasible $\bar{\gamma}=(\bar{t}, \bar{x})$ with $\bar{x}$ development efficient and define for each $v$,

$$
\bar{K}(v) \equiv \sum_{i} \bar{t}_{i}^{a}(v)-I \bar{x}_{0}(v) .
$$

Feasibility of $\bar{\gamma}$ means $\bar{x}(v)$ satisfies (3) for all $v \in V$, and therefore $\bar{K}(v) \geq 0$ for all $v \in V$. For any state $v$, let $\bar{n}(v) \equiv \sum_{i} \bar{x}_{i}(v)$ represent, for a given $v$, the total number of consumers with $x_{i}=1$. For any state $v$ with $\bar{x}_{0}(v)=0$, define $\hat{t}_{i}^{a}(v) \equiv 0$ and $\hat{t}_{i}^{p}(v) \equiv \bar{t}_{i}^{a}(v)+\bar{t}_{i}^{p}(v)$. For $\bar{x}_{0}(v)=1$ define $\hat{t}_{i}^{a}(v) \equiv \bar{t}_{i}^{a}(v)-\bar{x}_{i}(v) \bar{K}(v) / \bar{n}(v)$ and $\hat{t}_{i}^{p}(v) \equiv$ $\bar{t}_{i}^{p}(v)+\bar{x}_{i}(v) \bar{K}(v) / \bar{n}(v)$. Since $\bar{x}$ is feasible and development efficient, it holds $\bar{n}(v)>0$ if and only if $\bar{x}_{0}(v)=1$. Hence, the transformed transfer schedule $\hat{t}$ is well-defined. By construction, we have $\hat{t}_{i}^{a}(v)+\hat{t}_{i}^{p}(v)=\bar{t}_{i}^{a}(v)+\bar{t}_{i}^{p}(v)$ for all $v$, and $\sum_{i} \hat{t}_{i}^{a}(v)=0$ for any $v$ with $x_{0}(v)=0$, and $\sum_{i} \hat{t}_{i}^{a}(v)=\sum_{i} \bar{t}_{i}^{a}(v)-\bar{x}_{i}(v) \bar{K}(v) / \bar{n}(v)=\sum_{i} \bar{t}_{i}^{a}(v)-\bar{K}(v)=I$ for any $v$ with $\bar{x}_{0}(v)=1$. Hence, the allocation $(\hat{t}(v), \bar{x}(v))$ satisfies (12). Because the allocation $(\bar{t}(v), \bar{x}(v))$ is development feasible, also the allocation $(\hat{t}(v), \bar{x}(v))$ is development feasible. Moreover, from $\hat{t}_{i}^{a}(v)+\hat{t}_{i}^{p}(v)=\bar{t}_{i}^{a}(v)+\bar{t}_{i}^{p}(v)$ it follows that $(\hat{t}, \bar{x})$ is also budget-feasible, truthful, and individual rational, given that $(\bar{t}, \bar{x})$ is so by assumption. 
In order to show that $(\hat{t}, \bar{x})$ is feasible, it only remains to show that it is obedient, i.e., satisfies (8). To show this, define for $\bar{T}^{a} \in \overline{\mathcal{T}}^{a}$

$$
\bar{P}\left(\bar{T}^{a}\right)=\sum_{v \in \bar{V}\left(\bar{T}^{a}\right)} \pi(v)
$$

Now since, $\bar{\gamma}=(\bar{t}, \bar{x})$ is obedient by assumption, (8) holds for any $\bar{T}^{a} \in \overline{\mathcal{T}}^{a}$. Given that $\bar{T}^{a}=\sum_{i} \bar{t}_{i}^{a}(v)$ for any $v$ such that $\bar{\pi}\left(v \mid \bar{T}^{a}\right)>0$, we can rewrite (8) as

$$
\sum_{v \in V} \sum_{i=1}^{n} \bar{\pi}\left(v \mid \bar{T}^{a}\right)\left(\bar{t}_{i}^{p}(v)-c \bar{x}_{i}(v)+\bar{t}_{i}^{a}(v)\right)-I \geq \alpha \bar{T}^{a}, \text { for all } \bar{T}^{a} \in \overline{\mathcal{T}}^{a} .
$$

From $\hat{t}_{i}^{a}(v)+\hat{t}_{i}^{p}(v)=\bar{t}_{i}^{a}(v)+\bar{t}_{i}^{p}(v)$, this rewrites as

$$
\sum_{v \in V} \sum_{i=1}^{n} \bar{\pi}\left(v \mid \bar{T}^{a}\right)\left(\hat{t}_{i}^{p}(v)-c \bar{x}_{i}(v)+\hat{t}_{i}^{a}(v)\right)-I \geq \alpha \bar{T}^{a}, \text { for all } \bar{T}^{a} \in \overline{\mathcal{T}}^{a} .
$$

Because, by construction $\sum_{i} \hat{t}_{i}^{a}(v)=I$ for $v$ such that $\bar{\pi}\left(v \mid T^{a}\right)>0$, this rewrites as

$$
\sum_{v \in V} \sum_{i=1}^{n} \bar{\pi}\left(v \mid \bar{T}^{a}\right)\left(\hat{t}_{i}^{p}(v)-c \bar{x}_{i}(v)\right) \geq \alpha \bar{T}^{a}, \text { for all } \bar{T}^{a} \in \overline{\mathcal{T}}^{a} .
$$

Moreover, since feasibility implies that $\bar{T}^{a} \geq I$, the previous inequality implies that

$$
\sum_{v \in V} \sum_{i=1}^{n} \bar{\pi}\left(v \mid \bar{T}^{a}\right)\left(\hat{t}_{i}^{p}(v)-c \bar{x}_{i}(v)\right) \geq \alpha I, \text { for all } \bar{T}^{a} \in \overline{\mathcal{T}}^{a} .
$$

It follows after a further multiplication by $\bar{P}\left(\bar{T}^{a}\right)$ that

$$
\sum_{v \in V} \sum_{i=1}^{n} \bar{\pi}\left(v \mid \bar{T}^{a}\right) \bar{P}\left(\bar{T}^{a}\right)\left(\hat{t}_{i}^{p}(v)-c \bar{x}_{i}(v)\right) \geq \alpha I \cdot \bar{P}\left(\bar{T}^{a}\right), \text { for all } \bar{T}^{a} \in \overline{\mathcal{T}}^{a} .
$$

By definition of $\bar{\pi}\left(v \mid \bar{T}^{a}\right)$, we have $\bar{\pi}\left(v \mid \bar{T}^{a}\right) \bar{P}\left(\bar{T}^{a}\right)=\pi(v) \mathbf{1}_{v \in \bar{V}\left(\bar{T}^{a}\right)}$, where $\mathbf{1}_{A}$ is the indicator function which is 1 if the statement $A$ is true and 0 otherwise. Thus we may rewrite the former inequality as

$$
\sum_{v \in V} \sum_{i=1}^{n} \pi(v) \mathbf{1}_{v \in \bar{V}\left(\bar{T}^{a}\right)}\left(\hat{t}_{i}^{p}(v)-c \bar{x}_{i}(v)\right) \geq \alpha I \cdot \bar{P}\left(\bar{T}^{a}\right), \text { for all } \bar{T}^{a} \in \overline{\mathcal{T}}^{a}
$$

Summing over all $\bar{T}^{a} \in \overline{\mathcal{T}}^{a}$, we obtain

$$
\sum_{\bar{T}^{a} \in \overline{\mathcal{T}}^{a}} \sum_{v \in V} \sum_{i=1}^{n} \pi(v) \mathbf{1}_{v \in \bar{V}\left(\bar{T}^{a}\right)}\left(\hat{t}_{i}^{p}(v)-c \bar{x}_{i}(v)\right) \geq \sum_{\bar{T}^{a} \in \overline{\mathcal{T}}^{a}} \alpha I \cdot \bar{P}\left(\bar{T}^{a}\right) .
$$


Denoting by $\hat{V}(\cdot)$ and $\hat{P}(\cdot)$ under the mechanism $\hat{\gamma}$ the corresponding sets $\bar{V}(\cdot)$ and probabilities $\bar{P}(\cdot)$ under the mechanism $\bar{\gamma}$, we can, after noting that $\hat{\mathcal{T}}^{a}=\{I\}$ and $\hat{V}(I)=\left\{v \mid \bar{x}_{0}(v)=1\right\}=\cup_{\bar{T}^{a} \in \overline{\mathcal{T}}^{a}} \bar{V}\left(\bar{T}^{a}\right)$, rewrite the previous inequality as

$$
\sum_{v \in V} \sum_{i=1}^{n} \pi(v) \mathbf{1}_{v \in \hat{V}(I)}\left(\hat{t}_{i}^{p}(v)-c \bar{x}_{i}(v)\right) \geq \alpha I \cdot \bar{P}\left(\cup_{\bar{T}^{a} \in \overline{\mathcal{T}}^{a}} \bar{T}^{a}\right),
$$

which we can further rewrite as

$$
\sum_{v \in V} \sum_{i=1}^{n} \pi(v) \mathbf{1}_{\left\{\bar{x}_{0}(v)=1 \wedge \sum_{i} \hat{t}_{i}^{a}(v)=I\right\}}\left(\hat{t}_{i}^{p}(v)-c \bar{x}_{i}(v)\right) \geq \alpha I \cdot \hat{P}(I),
$$

but, since for $\hat{\gamma}$ we have $\hat{\mathcal{T}}^{a}=\{I\}$, this is equivalent to

$$
\sum_{v \in V} \sum_{i=1}^{n} \hat{\pi}\left(v \mid \hat{T}^{a}\right)\left(\hat{t}_{i}^{p}(v)-c \bar{x}_{i}(v)\right)-\hat{T}^{a}-I \geq \alpha I \cdot \hat{P}(I), \text { for all } \hat{T}^{a} \in \hat{\mathcal{T}}^{a} .
$$

Hence, $\hat{\gamma}$ satisfies (8) so that $\hat{\gamma}=(\hat{t}, \bar{x})$ is obedient. To complete the proof note that since $\hat{t}_{i}^{a}(v)+\hat{t}_{i}^{p}(v)=\bar{t}_{i}^{a}(v)+\bar{t}_{i}^{p}(v)$, the feasible direct mechanism $\hat{\gamma}=(\hat{t}, \bar{x})$ is payoff equivalent to original mechanism $\bar{\gamma}=(\bar{t}, \bar{x})$.

Q.E.D.

Proof of lemma 3: The first statement follows because the incentive constraint (7) for $v_{i}=1$, and the individual rationality (9) of a consumer with value $v=0$ imply the individual rationality (9) for $v_{i}=1$. That is, $1 \cdot X_{i}(1)-T_{i}(1) \geq 1 \cdot X_{i}(0)-T_{i}(0) \geq$ $0 \cdot X_{i}(0)-T_{i}(0) \geq 0$.

To see $x_{i}\left(0, v_{-i}\right)=0$, note that if not, then $x_{i}\left(0, v_{-i}\right)=1$. But then lowering it to 0 raises the objective $(15)$ by $p\left(0, v_{-i}\right) c$. This change is feasible, because it keeps constraints (7) for $v_{i}=0,(9)$, and (12) unaffected, while relaxing the constraints (7) for $v_{i}=1,(13)$, and (14). The statement $X_{i}(0)=0$ then follows as a corollary.

To see $T_{i}(0)=0$, note that $(9)$ implies $T_{i}(0) \leq 0$. But if $T_{i}(0)<0$, then raising each $t_{i}^{p}\left(0, v_{-i}\right)$ and $t_{i}^{p}\left(1, v_{-i}\right)$ by $T_{i}(0) / \pi_{i}\left(v_{-i}\right)$ for each $v_{-i} \in V_{-i}$ leads to a feasible mechanism with $T_{i}(0)=0$ and the same value for the objective (15). The adapted mechanism is feasible since the change does not affect (7) and (12), and, by construction, satisfies (9) for $v_{i}=0$ so that, by the first argument of this lemma, it also satisfies (9) for $v_{i}=1$. The raises in $t_{i}^{p}(v)$ further relaxes (13) and (14). Consequently, there is no loss of generality in assuming that, at the optimum, $T_{i}(0)=0$.

To see $T_{i}(1)=X_{i}(1)$, note that $(7)$ for $v_{i}=1$ together with $X_{i}(0)=T_{i}(0)=0$ imply $T_{i}(1) \leq X_{i}(1)$. But if $T_{i}(1)<X_{i}(1)$, then we can raise all $t_{i}^{p}\left(1, v_{-i}\right)$ by $\varepsilon>0$ 
such that $T_{i}(1)=X_{i}(1)$. The increase is feasible and does not affect the objective (15). To see that the change is feasible, note that it relaxes constraint (7) for $v_{i}=0$ and, by construction, satisfies (7) for $v_{i}=1$. It further does not affect (9) for $v_{i}=0$ and, by the first part of the lemma, the constraint (9) for $v_{i}=1$ is redundant. It also does not affect (12), while relaxing (13) and (14). Consequently, there is no loss of generality in assuming that, at the optimum, $T_{i}(1)=X_{i}(1)$.

Q.E.D.

Proof of Proposition 2: Recalling that $\pi^{*}=\sum_{v \in V^{1}} \pi(v)$, define

$$
\pi^{*}(v) \equiv \begin{cases}\pi(v) / \pi^{*} & \text { if } x_{0}^{*}(v)=1 \\ 0 & \text { otherwise }\end{cases}
$$

The proposition's condition $W^{*} \geq(1+\alpha) \pi^{*} I$ is then equivalent to

$$
\sum_{v \in V} \sum_{i} \pi^{*}(v) v_{i}(1-c) \geq(1+\alpha) I
$$

We first prove that under condition (44) the first best is implementable by constructing a transfer schedule $\hat{t}$ such that the direct mechanism $\gamma^{*}=\left(\hat{t}, x^{*}\right)$ is feasible and therefore implements $x^{*}$. For any $v$ such that $x_{0}^{*}(v)=0$, set $\hat{t}_{i}^{a}(v)=\hat{t}_{i}^{p}(v)=0$. For any $v$ such that $x_{0}^{*}(v)=1$, let $\bar{x}^{*}(v) \equiv \sum_{i} x_{i}^{*}(v)>0$ represents the efficient number of goods to be produced in state $v{ }^{29}$ Set $\hat{t}_{i}^{a}(v)=x_{i}^{*}(v) I / \bar{x}^{*}(v)$ and $\hat{t}_{i}^{p}(v)=x_{i}^{*}(v)\left(1-I / \bar{x}^{*}(v)\right)$.

We show that the resulting mechanism $\gamma^{*}=\left(\hat{t}, x^{*}\right)$ is direct and feasible. More specifically, for each $v \in V$ the allocation $\gamma^{*}(v)$ satisfies (3) so that $\gamma^{*}$ is direct (it trivially satisfies (4), since $x^{*}$ does so by construction). Moreover, the direct mechanism $\gamma^{*}$ satisfies (7), (8), and (9) for each $v \in V$.

To show (3) for $v$ such that $x_{0}^{*}(v)=0$, note that $\sum_{i} \hat{t}_{i}^{a}(v)=0=I x_{0}^{*}(v)$, and that $\sum_{i} \hat{t}_{i}^{a}(v)+\hat{t}_{i}^{p}(v)=0=I x_{0}^{*}(v)+c \sum_{i} x_{i}^{*}(v)$, since $x_{i}^{*}(v)=0$ for all $i$ whenever $x_{0}^{*}(v)=0$. To show (3) for $v$ such that $x_{0}^{*}(v)=1$, note that $\sum_{i} \hat{t}_{i}^{a}(v)=\sum_{i} x_{i}^{*}(v) I / \bar{x}^{*}(v)=I=$ $I x_{i}^{*}(0)$ and $\sum_{i} \hat{t}_{i}^{a}(v)+\hat{t}_{i}^{p}(v)=\sum_{\left\{i: x_{i}^{*}(v)=1\right\}}\left(\hat{t}_{i}^{a}(v)+\hat{t}_{i}^{p}(v)\right)+\sum_{\left\{i: x_{i}^{*}(v)=0\right\}}\left(\hat{t}_{i}^{a}(v)+\hat{t}_{i}^{p}(v)\right)=$ $\sum_{\left\{i: x_{i}^{*}(v)=1\right\}} 1+\sum_{\left\{i: x_{i}^{*}(v)=0\right\}} 0=\sum_{i} v_{i} \geq I+\sum_{i} c v_{i}=I x_{0}^{*}(v)+c \sum_{i} x_{i}^{*}(v)$, where the inequality holds because $x^{*}(0)=1$ is efficient by assumption so that $\sum_{i} v_{i} \geq$ $I+\sum_{i} c v_{i}$. Hence, $\gamma(v) \in A$ for all $v$ so that the mechanism $\gamma^{*}$ is direct.

\footnotetext{
${ }^{29} \bar{x}^{*}(v)$ is greater than 0 , since $x_{0}^{*}(v)=1$ and $x^{*}$ is development-efficient.
} 
To show (7) and (9) note that $x_{i}^{*}(0)=0$ implies $X_{i}^{*}(0)=0$ and, by construction of $\hat{t}$, also $T_{i}^{*}(0)=0$. Moreover, $X_{i}^{*}(1) \geq 0$ and $T_{i}^{*}(1) \geq 0$. For $v_{i}=0$, it therefore follows $v_{i} X_{i}^{*}\left(v_{i}\right)-T_{i}^{*}\left(v_{i}\right)=0 \cdot X_{i}^{*}(0)-T_{i}^{*}(0)=0 \leq-T_{i}^{*}(1)=0 \cdot X_{1}^{*}(1)-T_{i}^{*}(1)$ so that (7) and (9) are satisfied for $v_{i}=0$. To see that they are also satisfied for $v_{i}=1$, note that $1 \cdot X_{i}^{*}(1)-T_{i}^{*}(1)=\sum_{v_{-i}} \pi_{i}\left(v_{-i}\right)\left[x_{i}^{*}\left(1, v_{-i}\right)-\hat{t}_{i}^{a}\left(1, v_{-i}\right)-\hat{t}_{i}^{p}\left(1, v_{-i}\right)\right]=0=$ $1 \cdot X_{i}^{*}(0)-T_{i}^{*}(0)$.

Finally, to show (8), first note that for $\gamma^{*}$ we have $\mathcal{T}^{*}=\{I\}$ and $\pi(v \mid I)=\pi^{*}(v)$ so that we only need to show $\sum_{v \in V} \sum_{i=1}^{n} \pi^{*}(v)\left[\hat{t}_{i}^{p}(v)-c x_{i}^{*}(v)\right] \geq \alpha I$, which follows from

$$
\begin{aligned}
& \sum_{v \in V} \sum_{i=1}^{n} \pi^{*}(v)\left[\hat{t}_{i}^{p}(v)-c x_{i}^{*}(v)\right]=\sum_{i=1}^{n} \sum_{v: x_{i}^{*}(v)=1} \pi^{*}(v)\left[1-I / \bar{x}^{*}(v)-c\right]= \\
& =\sum_{i=1}^{n} \sum_{v: x_{i}^{*}(v)=1} \pi^{*}(v)(1-c)-I=\sum_{i=1}^{n} \sum_{v \in V} \pi^{*}(v) v_{i}(1-c)-I \geq \alpha I,
\end{aligned}
$$

where the inequality uses (44).

We next show that if condition (44) is violated so that

$$
\sum_{v \in V} \sum_{i} \pi^{*}(v) v_{i}(1-c)<(1+\alpha) I
$$

then there does not exist a transfer schedule $\hat{t}$ such that the direct mechanism $\gamma=$ $\left(\hat{t}, x^{*}\right)$ is feasible. In particular, we show there does not exist a transfer schedule $\hat{t}$ such that $\left(\hat{t}, x^{*}\right)$ satisfies $(16)-(22)$.

For the efficient output schedule $x^{*}$ it holds $V^{1}=\left\{v \mid x_{0}^{*}(v)=1\right\}$ and $V^{0}=$ $\left\{v \mid x_{0}^{*}(v)=0\right\}$ and $V=V^{1} \cup V^{0}$.

For $v \in V^{0}$, it therefore holds $x_{i}(v)=0$ so that conditions (19) and (20) taken together imply $\sum_{i} t_{i}^{a}(v)+t_{i}^{p}(v) \geq 0$. Multiplying by $\pi(v)$ and summing up over all $v$ in $V^{0}$ yields

$$
\sum_{v \in V^{0}} \sum_{i} \pi(v)\left[t_{i}^{a}(v)+t_{i}^{p}(v)\right] \geq 0
$$

For $v \in V^{1}$, (19) implies $\sum_{i} t_{i}^{a}(v)=I$. Multiplying by $\pi(v)$ and summing up over all $v$ in $V^{1}$ yields

$$
\sum_{v \in V^{1}} \sum_{i} \pi(v) t_{i}^{a}(v)=\sum_{v \in V^{1}} \pi(v) I=\pi^{*} I
$$


Since (19) implies $\mathcal{T}^{a}=\{I\}$, it follows that $\pi^{*} \cdot \pi(v \mid I)=\pi(v)$ for $v \in V^{1}$ and $\pi(v \mid I)=0$ for all $v \in V^{0}$. Hence, after a multiplication by $\pi^{*}$ we can rewrite (17) as

$$
\sum_{v \in V^{1}} \sum_{i} \pi(v) t_{i}^{p}(v) \geq \alpha \pi^{*} I+\sum_{v \in V^{1}} \sum_{i} \pi(v) c x_{i}^{*}(v)
$$

Combining (49) and (50) yields

$$
\sum_{v \in V^{1}} \sum_{i} \pi(v)\left[t_{i}^{a}(v)+t_{i}^{p}(v)\right] \geq(1+\alpha) \pi^{*} I+\sum_{v \in V^{1}} \sum_{i} \pi(v) c x_{i}^{*}(v)
$$

Since $x_{i}^{*}(v)=0$ for $v \in V^{0},(51)$ together with (48) imply

$$
\sum_{v \in V} \sum_{i} \pi(v)\left[t_{i}^{a}(v)+t_{i}^{p}(v)\right] \geq(1+\alpha) \pi^{*} I+\sum_{v \in V} \sum_{i} \pi(v) c x_{i}^{*}(v)
$$

Since $x_{i}^{*}(v)=v_{i}$ for $v \in V^{1}$ and $x_{i}^{*}(v)=0$ for $v \in V^{0}$, multiplying (47) by $\pi^{*}$ and rearranging terms yields

$$
(1+\alpha) \pi^{*} I+\sum_{v \in V} \sum_{i} \pi(v) c x_{i}^{*}(v)>\sum_{v \in V} \sum_{i} \pi(v) x_{i}^{*}(v)
$$

Combining this latter inequality with inequality (52) yields

$$
\sum_{v \in V} \sum_{i} \pi(v)\left[t_{i}^{a}(v)+t_{i}^{p}(v)\right]>\sum_{v \in V} \sum_{i} \pi(v) x_{i}^{*}(v)
$$

Condition (16) implies after multiplying by $p_{i}(1)$ and summing over all $i$

$$
\sum_{i} \sum_{v_{-i}} p\left(1, v_{-i}\right)\left[t_{i}^{a}\left(1, v_{-i}\right)+t_{i}^{p}\left(1, v_{-i}\right)\right]=\sum_{i} \sum_{v_{-i}} p\left(1, v_{-i}\right) x_{i}^{*}\left(1, v_{-i}\right) .
$$

Similarly, (18) implies after multiplying by $p_{i}(0)$ and summing over all $i$

$$
\sum_{i} \sum_{v_{-i}} p\left(0, v_{-i}\right)\left[t_{i}^{a}\left(0, v_{-i}\right)+t_{i}^{p}\left(0, v_{-i}\right)\right]=0=\sum_{i} \sum_{v_{-i}} p\left(0, v_{-i}\right) x_{i}^{*}\left(0, v_{-i}\right)
$$

because $x_{i}^{*}\left(0, v_{-i}\right)=0$.

Combining the latter two inequalities yields

$$
\sum_{i} \sum_{v \in V} \pi(v)\left[t_{i}^{a}(v)+t_{i}^{p}(v)\right]=\sum_{i} \sum_{v \in V} \pi(v) x_{i}^{*}(v)
$$

but this contradicts (54). Hence, under (47) there does not exist a direct mechanism $\gamma=\left(t, x^{*}\right)$ that satisfies (16)-(22) and, hence, $x^{*}$ is not implementable.

Q.E.D. 
Proof of Proposition 3: Consider a maximizer $\bar{\gamma}=(\bar{t}, \bar{x})$ of problem $\mathcal{P}$.

To show that it satisfies the first statement, note that (22) directly implies that for $v_{i}=0$ it holds $x_{i}\left(v_{i}, v_{-i}\right)=v_{i}$. So it is left to prove $x_{0}^{s b}\left(1, v_{-i}\right)=1 \Rightarrow \bar{x}_{i}\left(1, v_{-i}\right)=1$. Suppose to the contrary that there exists some $\bar{v} \in V$ with some $\bar{v}_{i}=1$ so that $\bar{x}_{0}(\bar{v})=1$ and $\bar{x}_{i}\left(1, \bar{v}_{-i}\right)=0$. Then by raising both $\bar{x}_{i}\left(1, \bar{v}_{-i}\right)$ and the corresponding $\bar{t}_{i}^{p}\left(1, \bar{v}_{-i}\right)$ by 1 , the objective $(15)$ is raised by $p\left(1, \bar{v}_{-i}\right)(1-c)>0$, while the constraints (16), (18),(19),(21), and (22) are unaffected, and (17) and (20) are relaxed.

To show the second statement, suppose to the contrary that $\bar{\gamma}=(\bar{t}, \bar{x})$ exhibits $\bar{x}_{0}(\bar{v})=1$, while $x_{0}^{*}(\bar{v})=0$ for some $\bar{v}=\left(\bar{v}_{1}, \ldots, \bar{v}_{n}\right)$. Define $I^{1}=\left\{i \mid \bar{x}_{i}(\bar{v})=1\right\}$ as the set of consumers who receive the good under $\bar{\gamma}$ and the value realization $\bar{v}$. Since $\bar{\gamma}$ is, by assumption, a maximizer of $\mathcal{P}$, it must hold that $I^{1}$ is non-empty and, due to (22), for all $i \in I^{1}$ it holds $v_{i}=1$. But since $x_{0}^{*}(v)=0$, it follows $\sum_{i \in I^{1}} \bar{v}_{i}(1-c) \leq \sum_{i} \bar{v}_{i}(1-c)<I$. Now consider an alternative mechanism $\hat{\gamma}=(\hat{t}, \hat{x})$ that is identical to $\bar{\gamma}$ except that $\hat{x}_{0}(\bar{v})=\hat{x}_{i}(\bar{v})=0$ and for all $i \in I^{1}$ it exhibits $\hat{x}_{i}(\bar{v})=0, \hat{t}_{i}^{p}(\bar{v})=\bar{t}_{i}^{p}(\bar{v})-c$, and $\hat{t}_{i}^{a}(\bar{v})=\bar{t}_{i}^{a}(\bar{v})-1+c$. First note that a comparison of the objective (15) evaluated at $\hat{\gamma}$ and $\bar{\gamma}$ yields a difference of $p(\bar{v})\left[I-\sum_{i \in I^{1}}(1-c)\right]$, which is positive. Hence, $\bar{\gamma}$ is not a solution to $\mathcal{P}$ if $\hat{\gamma}$ is feasible. In order to see that $\hat{\gamma}$ is feasible, we verify that it satisfies (3), (4), (7), (8), and (9) using that $\bar{\gamma}$ satisfies these constraints by assumption.

To verify the first inequality in (3), note $\sum_{i} \hat{t}_{i}^{a}(\bar{v})=\sum_{i \in I^{1}}\left(\bar{t}_{i}^{a}(\bar{v})-1+c\right)+$ $\sum_{i \notin I^{1}} \bar{t}_{i}^{a}(\bar{v}) \geq I-\sum_{i \in I^{1}}(1-c) \geq 0=I \hat{x}_{0}(\bar{v})$, where the first inequality follows because $\bar{\gamma}$ satisfies (3) and the second inequality was already established above.

To verify the second inequality in (3) note $\sum_{i}\left(\hat{t}_{i}^{a}(\bar{v})+\hat{t}_{i}^{p}(\bar{v})\right)=\sum_{i \in I^{1}}\left(\bar{t}_{i}^{a}(\bar{v})+\right.$ $\left.\bar{t}_{i}^{p}(\bar{v})-1\right)+\sum_{i \notin I^{1}}\left(\bar{t}_{i}^{a}(\bar{v})+\bar{t}_{i}^{p}(\bar{v})\right) \geq I+c \sum_{i \in I^{1}} \bar{x}_{i}(\bar{v})-\sum_{i \in I^{1}} 1=I-\sum_{i \in I^{1}}(1-c) \geq$ $0=I \hat{x}_{0}(\bar{v})+c \sum_{i} \hat{x}_{i}(\bar{v})$, where the first inequality follows because $\bar{\gamma}$ satisfies (3).

Noting that, because $\bar{\gamma}$ satisfies (4), it trivially follows that also $\hat{\gamma}$ satisfies (4), we continue to verify (7) and (9). Note that, by construction, $\hat{x}_{i}(\bar{v})-\hat{t}_{i}^{a}(\bar{v})-\hat{t}_{i}^{p}(\bar{v})=$ $\bar{x}_{i}(\bar{v})-\bar{t}_{i}^{a}(\bar{v})-\bar{t}_{i}^{p}(\bar{v})$ so that $\hat{X}_{i}\left(v_{i}\right)-\hat{T}_{i}\left(v_{i}\right)=\bar{X}_{i}\left(v_{i}\right)-\bar{T}_{i}\left(v_{i}\right)$. Because $\bar{\gamma}$ satisfies $(7)$ and (9), therefore, also $\hat{\gamma}$.

Finally, to verify (8) note that for $\bar{\gamma}$ we have $\mathcal{T}^{a}=\{I\}$ so that this is also the case for $\hat{\gamma}$. Hence, (8) reduces to (18). To see that $\hat{\gamma}$ satisfies this constraint, note that $\sum_{v \in V} \sum_{i=1}^{n} \pi(v \mid I)\left(\hat{t}_{i}^{p}(v)-c \hat{x}_{i}(v)\right)=\sum_{v \neq \bar{v}} \sum_{i=1}^{n} \pi(v \mid I)\left(\hat{t}_{i}^{p}(v)-c \hat{x}_{i}(v)\right)+$ 


$$
\begin{aligned}
& \sum_{i} p(\bar{v} \mid I)\left(\hat{t}_{i}^{p}(\bar{v})-c \hat{x}_{i}(\bar{v})\right)=\sum_{v \neq \bar{v}} \sum_{i} \pi(v \mid I)\left(\bar{t}_{i}^{p}(v)-c \bar{x}_{i}(v)\right)+\sum_{i} p(\bar{v} \mid I) \hat{t}_{i}^{p}(\bar{v})=\sum_{v \neq \bar{v}} \sum_{i} \pi(v \mid I)\left(\bar{t}_{i}^{p}(v)-\right. \\
& \left.c \bar{x}_{i}(v)\right)+\sum_{i \notin I^{1}} p(\bar{v} \mid I) \hat{t}_{i}^{p}(\bar{v})+\sum_{i \in I^{1}} p(\bar{v} \mid I) \hat{t}_{i}^{p}(\bar{v})=\sum_{v \neq \bar{v}} \sum_{i} \pi(v \mid I)\left(\bar{t}_{i}^{p}(v)-c \bar{x}_{i}(v)\right)+ \\
& \sum_{i \notin I^{1}}^{n} p(\bar{v} \mid I) \bar{t}_{i}^{p}(\bar{v})+\sum_{i \in I^{1}}^{n} p(\bar{v} \mid I)\left(\bar{t}_{i}^{p}(\bar{v})-c\right)=\sum_{v \neq \bar{v}} \sum_{i} \pi(v \mid I)\left(\bar{t}_{i}^{p}(v)-c \bar{x}_{i}(v)\right)+\sum_{i \notin I^{1}}^{n} p(\bar{v} \mid I)\left(\bar{t}_{i}^{p}(\bar{v})-\right. \\
& \left.c \bar{x}_{i}(\bar{v})\right)+\sum_{i \in I^{1}}^{n} p(\bar{v} \mid I)\left(\bar{t}_{i}^{p}(\bar{v})-c \bar{x}_{i}(\bar{v})\right)=\sum_{v \in V} \sum_{i} \pi(v \mid I)\left(\bar{t}_{i}^{p}(v)-c \bar{x}_{i}(v)\right) \geq \alpha I .
\end{aligned}
$$

To show the proposition's third statement, consider a mechanism $\bar{\gamma}=(\bar{x}, \bar{t})$ which satisfies (16)-(22) and there is a $\bar{v}$ such that $\bar{x}_{0}(\bar{v})=0$, while $\sum_{i} \bar{v}_{i}>(1+\alpha) I /(1-c)$. We show that $\bar{\gamma}$ is not a solution to $\mathcal{P}$, because there exists a $(\hat{x}, \hat{t})$ that also satisfies (16)-(22) but yields a strictly higher surplus that $\bar{\gamma}$. More specifically, let $(\hat{x}, \hat{t})$ be identical to $(\bar{x}, \bar{t})$ except that $\hat{x}_{i}(\bar{v})=\bar{v}_{i}, \hat{t}_{i}^{a}(\bar{v})=\bar{t}_{i}^{a}(\bar{v})+\bar{v}_{i} \cdot I / \sum_{j} \bar{v}_{j}$, and $\hat{t}_{i}^{p}(\hat{v})=$ $\tilde{t}_{i}^{p}(\hat{v})+\bar{v}_{i}\left(1-I / \sum_{j} \bar{v}_{j}\right)$.

Note first that the difference in surplus between $(\hat{x}, \hat{t})$ and $(\bar{x}, \bar{t})$ is $p(\bar{v})[(1-$ c) $\left.\sum_{j} v_{j}-I\right]>0$. It remains to be checked that $(\hat{x}, \hat{t})$ satisfies (16)-(22). That it satisfies (16), (18), (21), and (22) follows directly, because $(\bar{x}, \bar{t})$ satisfies these constraints by assumption and $(\hat{x}, \hat{t})$ is a transformation of $(\bar{x}, \bar{t})$ which preserves them.

Since $(19)$ holds for $(\bar{x}, \bar{t}),(\hat{x}, \hat{t})$ trivially satisfies it for all $v \neq \hat{v}$. It, however, also holds for $\hat{v}$, since $\sum_{i} \hat{t}_{i}^{a}(\bar{v})=\sum_{i} \bar{t}_{i}^{a}(\bar{v})+\sum_{i} \bar{v}_{i} \cdot I / \sum_{j} \bar{v}_{j}=I \bar{x}_{0}(\bar{v})+I=I=I \hat{x}_{0}(\bar{v})$. Similarly (20), holds for all $v \neq \bar{v}$, while for $\bar{v}$ it follows $\sum_{i} \hat{t}_{i}^{p}(\bar{v})=\sum_{i} \bar{t}_{i}^{p}(\bar{v})+$ $\sum_{i} \bar{v}_{i}\left(1-I / \sum_{j} \bar{v}_{j}\right) \geq \sum_{i} \hat{x}_{i}(\bar{v})\left(1-I / \sum_{j} \bar{v}_{j}\right)>\sum_{i} \hat{x}_{i}(\bar{v}) c$, where the first inequality uses that $(\bar{x}, \bar{t})$ satisfies $(20)$, and the second inequality follows from the proposition's presumption that $\sum_{j} \bar{v}_{j}>(1+\alpha) I /(1-c)$, as this implies $c<1-I / \sum_{j} \bar{v}_{j}$.

Finally, to see that $(\hat{x}, \hat{t})$ satisfies $(17)$ because $(\bar{x}, \bar{t})$ does so, first define

$$
\bar{R}(v)=\sum_{i}\left[\bar{t}_{i}^{p}(v)-c \bar{x}_{i}(v)\right] \text { and } \hat{R}(v)=\sum_{i}\left[\hat{t}_{i}^{p}(v)-c \hat{x}_{i}(v)\right]
$$

It holds $\bar{R}(v)=\hat{R}(v)$ for all $v \neq \bar{v}$, while for $\bar{v}$ it follows $\hat{R}(\bar{v})=\sum_{i}\left[\hat{t}_{i}^{p}(\bar{v})-c \hat{x}_{i}(\bar{v})\right]=$ $\sum_{i}\left[\bar{t}_{i}^{p}(\bar{v})+\bar{v}_{i}\left(1-I / \sum_{j} \bar{v}_{j}\right)-c \bar{v}_{i}\right] \geq \sum_{i} \bar{v}_{i}\left(1-I / \sum_{j} \bar{v}_{j}-c\right)=\sum_{i} \bar{v}_{i}(1-c)-I>\alpha I$, where the first inequality uses that $(\bar{x}, \bar{t})$ satisfies $(20)$, and the final inequality uses the proposition's presumption that $\sum_{i} \bar{v}_{i}>(1+\alpha) I /(1-c)$.

Since $(\bar{x}, \bar{t})$ satisfies $(17)$, the definition of $\pi(v \mid I)$ implies that it holds

$$
\sum_{\left\{v: \bar{x}_{0}(v)=1\right\}} \pi(v) \bar{R}(v) \geq \alpha I \cdot \sum_{\left\{v: \bar{x}_{0}(v)=1\right\}} \pi(v) .
$$


Combining this with the previously established inequality $\hat{R}(\bar{v})>\alpha I$, it follows

$$
p(\bar{v}) \hat{R}(\bar{v})+\sum_{\left\{v: \bar{x}_{0}(v)=1\right\}} \pi(v) \bar{R}(v) \geq \alpha I\left[p(\bar{v})+\sum_{\left\{v: \bar{x}_{0}(v)=1\right\}} \pi(v)\right] .
$$

But since $\left\{v: \hat{x}_{0}(v)=1\right\}=\left\{v: \bar{x}_{0}(v)=1\right\} \cup\{\bar{v}\}$, this is equivalent to

$$
\sum_{\left\{v: \hat{x}_{0}(v)=1\right\}} \pi(v) \hat{R}(v) \geq \alpha I \cdot \sum_{\left\{v: \hat{x}_{0}(v)=1\right\}} \pi(v),
$$

which is equivalent to saying that $(\hat{x}, \hat{t})$ satisfies $(17)$.

To show the proposition's last statement, suppose that, to the contrary, there exists a solution $\bar{\gamma}=(\bar{x}, \bar{t})$ of problem $\mathcal{P}$ for which such a $T$ does not exist. In this case, there exist a valuation profile $\bar{v}$ and $\hat{v}$ with $\sum_{j} \bar{v}_{j}>\sum_{j} \hat{v}_{j}$ such that $\bar{x}_{0}(\bar{v})=0$ and $\bar{x}_{0}(\hat{v})=1$. Since $\sum_{j} \bar{v}_{j}>\sum_{j} \hat{v}_{j}$, we can find a bijective correspondence $k:\{1, \ldots, n\} \rightarrow\{1, \ldots, n\}$ such that $\hat{v}_{j}=1$ implies $\bar{v}_{k(j)}=1$. Fix the correspondence $k$ and its inverse $k^{-1}$ and define the mechanism $\hat{\gamma}=(\hat{x}, \hat{t})$ by $\hat{x}_{0}(v)=\bar{x}_{0}(v)$ for all $v \neq \hat{v}, \bar{v}, \hat{x}_{0}(\bar{v})=1$ and $\hat{x}_{0}(\hat{v})=0$, and $\hat{x}_{i}(v)=\bar{x}_{k^{-1}(i)}(v), \hat{t}_{i}^{a}(v)=\bar{t}_{k^{-1}(i)}^{a}(v)$, $\hat{t}_{i}^{p}(v)=\bar{t}_{k^{-1}(i)}^{p}(v)$. Since $\bar{\gamma}$ satisfies by assumption all constraints (16)-(22) of problem $\mathcal{P}$, so does $\hat{\gamma}$. They also yield the same objective (15). But since $\sum_{j} \bar{v}_{j}>\sum_{j} \hat{v}_{j}$, mechanism $\hat{\gamma}$ exhibits at least one $i$ such that $\bar{v}_{i}=1$ and $x_{i}(\bar{v})=0$. By the first statement of this proposition, $\hat{\gamma}$ is not optimal, since there exists a feasible $\tilde{\gamma}$ which yields a strictly larger surplus. Consequently, we obtain the contradiction that $\bar{\gamma}$ is not optimal.

Q.E.D. 


\section{References}

Agrawal, A., C. Catalini, and A. Goldfarb, (2011). "The geography of crowdfunding," NBER Working Paper 16820.

Agrawal, A., C. Catalini, and A. Goldfarb, (2014) "Some Simple Economics of Crowdfunding," Innovation Policy and the Economy 14, 63-97.

Barbieri, S. and Malueg, D. (2010). "Prot-Maximizing Sale of a Discrete Public Good via the Subscription Game in Private-Information Environments" The B.E. Journal of Theoretical Economics 10, Article 5.

Belleflamme, P., T. Lambert, and A. Schwienbacher (2014). "Crowdfunding: Tapping the right crowd," Journal of Business Venturing 29, 585-609.

Belleflamme, P., N. Omrani, and M. Peitz (2015). "The economics of crowdfunding platforms", Information Economics and Policy 33, 1128.

Bergemann, D., B. Brooks, and S. Morris (2015). "The Limits of Price Discrimination." American Economic Review, 105, 921-57.

Chang, J.-W., (2015) "The Economics of Crowdfunding", mimeo University of California, Los Angeles.

Cornelli, F. (1996). "Optimal Selling Procedures with Fixed Costs," Journal of Economic Theory 71, 1-30.

Cowley, S., D. Goldman, J. Pepitone, L. Segall, O. Smith (2012). "Kickstarter's Top 50 Projects: When They Shipped," CNNMoney.

Dana, J., (1999). "Equilibrium price dispersion under demand uncertainty: The roles of costly capacity and market structure," Rand Journal of Economics 30, 632-660.

Deneckere, R. and J. Peck (1995). "Competition over Price and Service Rate when Demand Is Stochastic: A Strategic Analysis," Rand Journal of Economics 26, 148-161. 
Diamond, D., (1984). "Financial Intermediation and Delegated Monitoring," Review of Economic Studies 51, 393-414.

Ding, M., (2007). “An Incentive-Aligned Mechanism for Conjoint Analysis," Journal of Marketing Research 44, 214223

Dingman, S., (2013). "Canadian's smartwatch startup matches record \$15-million in VC funding" The Globe and Mail 16.5.2013, http://www.theglobeandmail. com/technology/business-technology/canadians-smartwatch-startup-matches-recordarticle11965214/, last retrieved 25 July 2015.

Ellman, M. and S. Hurkens (2014). "Optimal Crowdfunding Design", mimeo Institute for Economic Analysis (CSIC) and Barcelona GSE.

Hakenes, H., and F. Schlegel (2015) "Exploiting the Financial Wisdom of the Crowd - Crowdfunding as a Tool to Aggregate Vague Information", mimeo Bonn University.

Hildebrand, T., M. Puri, and J. Rocholl, (2013). "Adverse incentives in crowdfunding," Working Paper ESMT Berlin.

Jovanovic, B. and R. Rob, (1987). "Demand-driven innovation and spatial competition over time," Review of Economic Studies 54, 6372.

Klemperer, P. and M. Meyer, (1989). "Supply Function Equilibria in Oligopoly Under Uncertainty," Econometrica 89 1243-1277.

Kuppuswamy, V., and B. Bayus, (2013). "Crowdfunding creative ideas: The dynamics of project backers in Kickstarter," mimeo, University of North Carolina.

Lauga, D., and E. Ofek, (2009). "Market Research and Innovation Strategy in Duopoly," Marketing Science 28(2), 373-396.

Mollick, E., (2014). "The dynamics of crowdfunding: Determinants of success and failures," Journal of Business Venturing 29, 1-16.

Myerson, R., (1982). "Optimal coordination mechanism in generalized principalagent problems", Journal of Mathematical Economics 10, 6781. 
SEC, (2015). "SEC Adopts Rules to Facilitate Smaller Companies Access to Capital New Rules Provide Investors With More Investment Choices", http://www . sec.gov/news/pressrelease/2015-49.html, last retrieved 20 July 2015.

Strausz, R. (2005). "Honest Certification and the Threat of Capture", International Journal of Industrial Organization 23, 45-62.

The Economist, (2010). "The micro-price of micropatronage", 27.9.2010, http:// WwW.economist.com/blogs/babbage/2010/09/micropatronage_sweet_spot? fsrc=nwl, last retrieved 22 July 2015.

The Economist, (2012). "Raising capital online - The new thundering her," 16.06.2012, http://www. economist.com/node/21556973, last retrieved 20 July 2015.

Time, (2010). "The 50 Best Inventions of 2010" 11.11.2010, http://content.time. com/time/specials/packages/article/0, 28804, 2029497_2030652_2029823, 00.html, last retrieved 20 July 2015. 